\title{
ABSTRACT
}

\section{Mortality rate and years of life lost due to cancer in Iran from 2011 to 2018}

\author{
Mohammad Torkashvand Moradabadi ${ }^{1}$, Wahed Soroush ${ }^{1}$, Zahra Torkashvand $^{2 *}$ \\ 1. Faculty of Social Sciences, Yazd University, Yazd, Iran \\ 2. School of Paramedicine, Iran University of Medical Sciences, Tehran
}

Received: 22 April 2021

Accepted for publication: 16 May 2021

[EPub a head of print-28 June 2021]

Payesh: 2021; 20 (3): 333-345

Objective (s): Cancer is one of the leading causes of death. Cancer is the third leading cause of death in Iran. The goal of this study was to identify the trends in cancer mortality by age and sex in Iran from 2011 to 2018.

Methods: We analyzed cancer mortality data available in the Civil Registration Organization. A total of 278301 cancer deaths were registered in this database during 2011-2018. The rate of cancer deaths was then compared by age, sex, and time.

Results: The cancer death rate (per 100,000) has increased from 38 in 2011 to 47 in 2017 . The mean age of mortality by cancer, death rate, and its increase was higher among men than women. Premature death due to cancer has increased from 420 to 530 thousand person-years of life during 2011 to 2016, respectively. This increase was mainly due to an increase in adulthood cancer and old age, especially among the male gender.

Conclusion: Cancer deaths have increased in Iran since cancer has increased in the country as an epidemiological transition consequence. To overcome such excess mortality preventive measures are recommended.

Key Words: Demographic Transition, Death, Cancer, Years of Life Lost, YLL, Iran

\footnotetext{
* Corresponding author: School of Paramedicine, Iran University of Medical Sciences, Tehran

E-mail: z.torkashvand10@gmail.com
} 


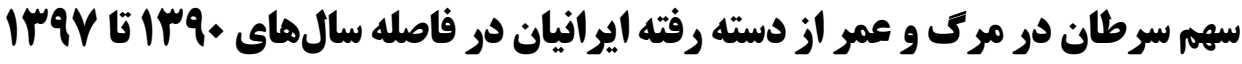

\author{
محمد تر كاشوند مرادآبادى'، واحد سروش'، زهرا تر كاشوند' \\ ا. ا.نشكده علوم اجتماعى، دانشكاه يزد، يزد، ايران

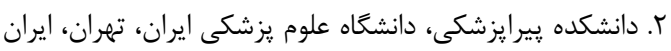

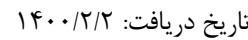

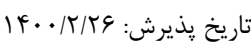

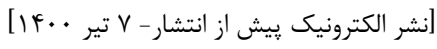

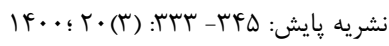

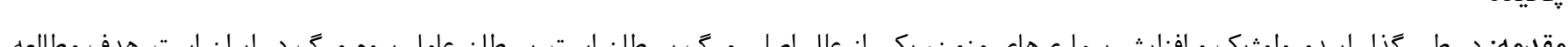

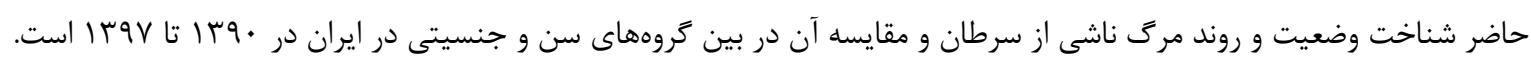

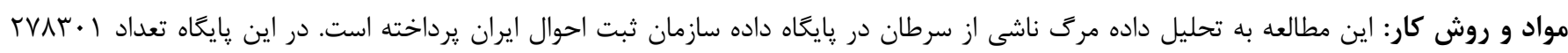

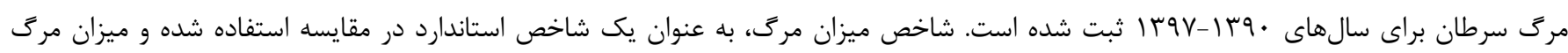

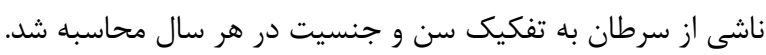

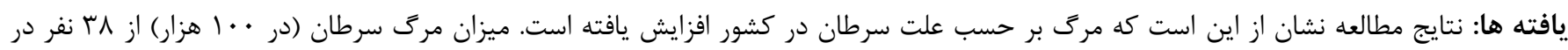

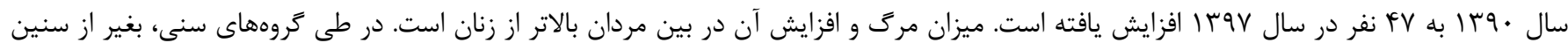

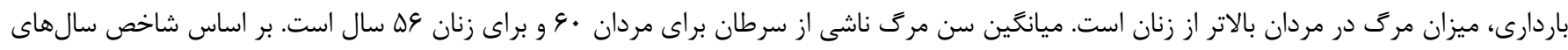

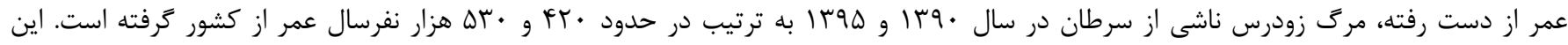

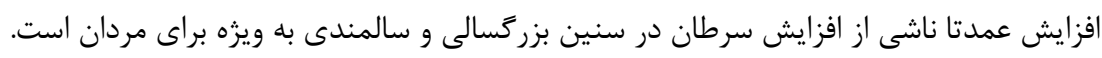

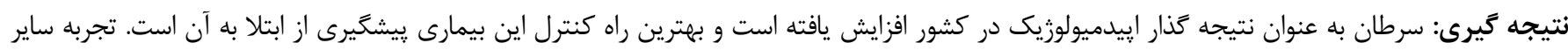

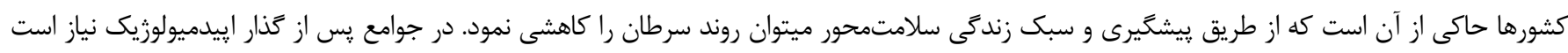

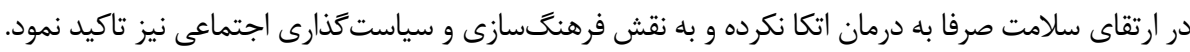

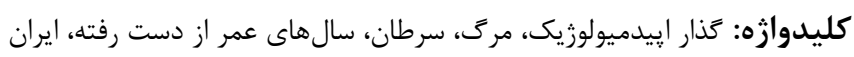


به سرطان است كه اين امر باعث شده است كه سرطان را به دومين

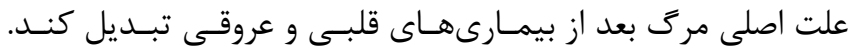

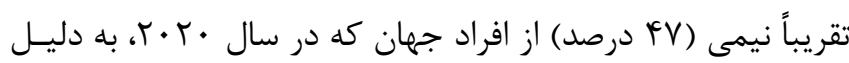

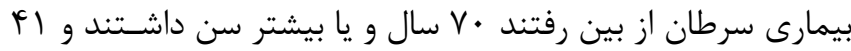

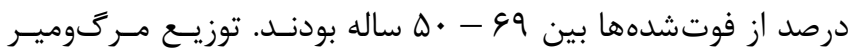

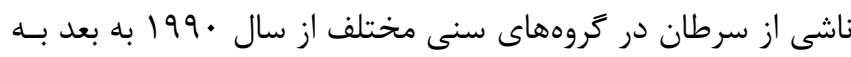

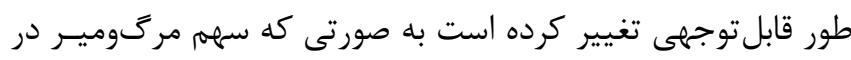

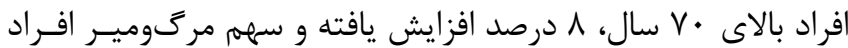

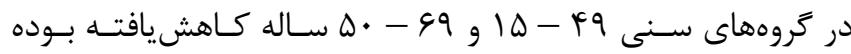

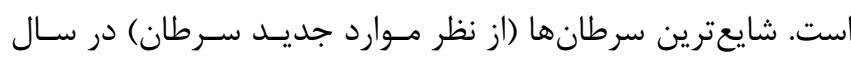

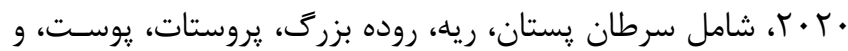

معده است [9،

بر اساس كزارش سازمان سـلامت جهـان در سـال 9 أ با، سـرطان

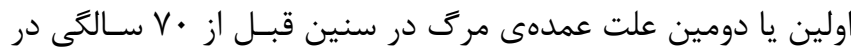

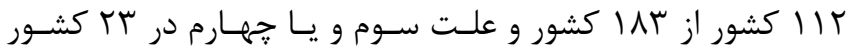

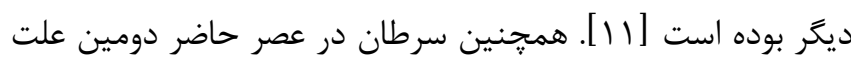

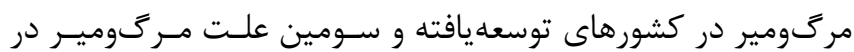

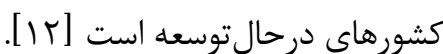
در ايران مركَومير ناشى از سرطان در رتبه سوم علل مركى و يس از

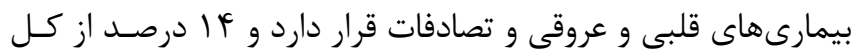

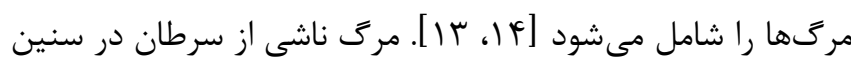

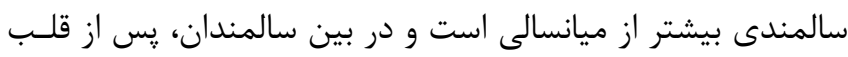

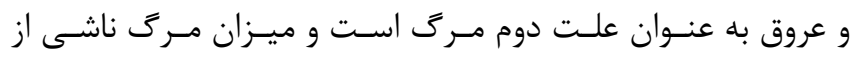

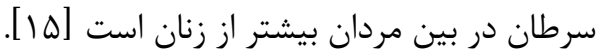

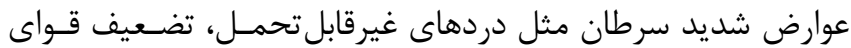

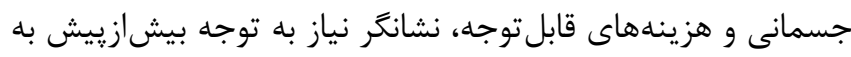

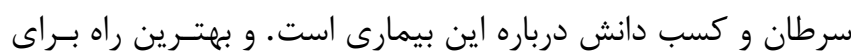

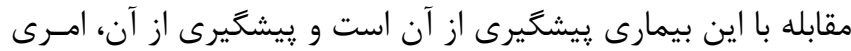

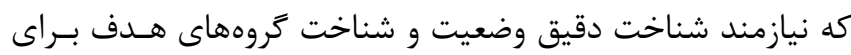

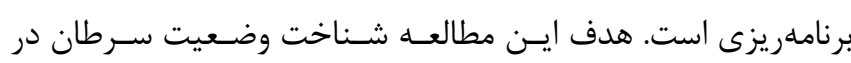

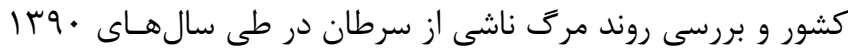

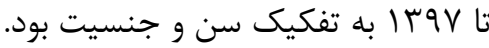

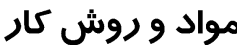

يافته هاى تحقيق حاضر بر اساس تحليل دادههاى ثانويه انجام شـده

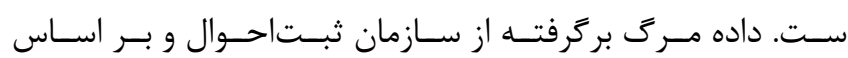

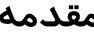

آمار مرك بر حسب علت، نقـش تعيسين كنــــه در تعيـين وضـعيت

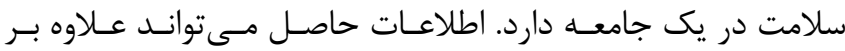

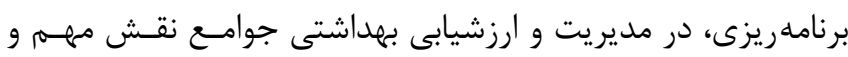

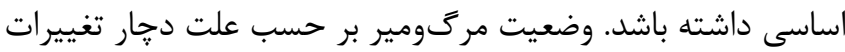

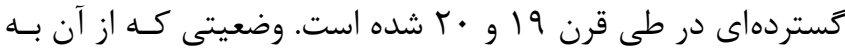

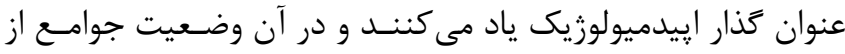

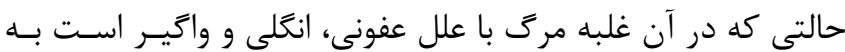
وضعيت مرك با علل غيرعفونى، مزمن و غيرواگير تغيير پِيدا مى كند

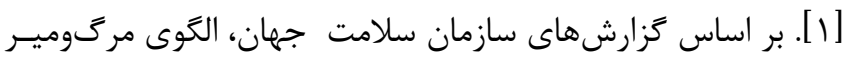

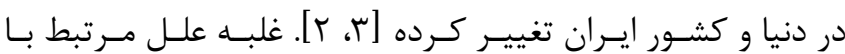
بيمارى هاى عفونى و انتلى در نيمه اول قرن بيستمه در ميـان علـل

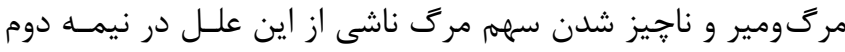

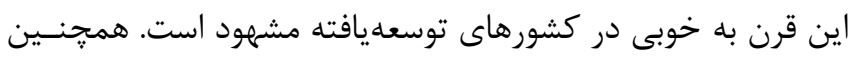

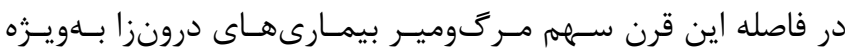

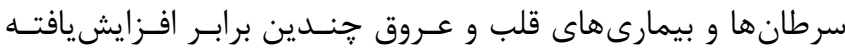

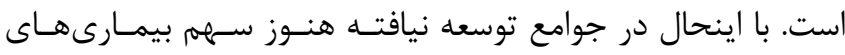

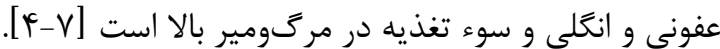

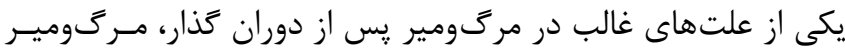

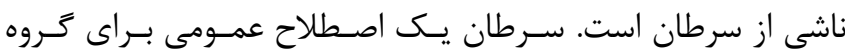

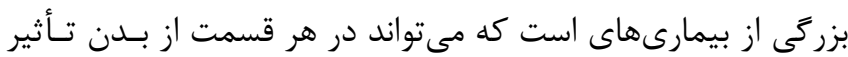

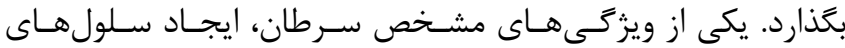

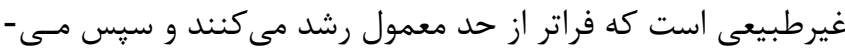

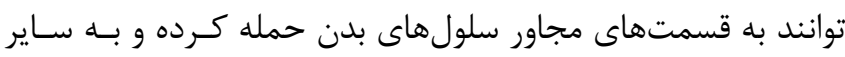

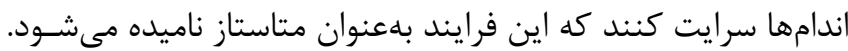

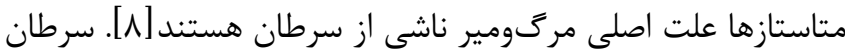

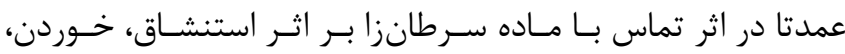

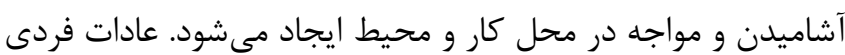

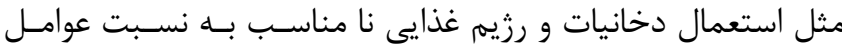

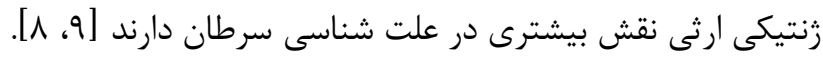

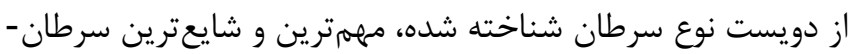

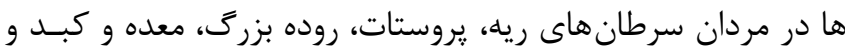

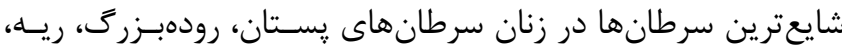

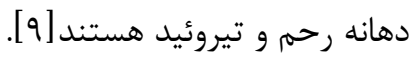

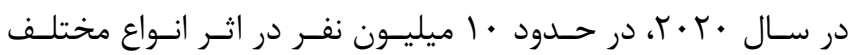
سرطان در دنيا فوت كردهاند. از هر شش مرى، يك مركى آن مربوط 
كشور رخ داده است. مرك ناشى از سرطان در كشور رو بــه افزايش

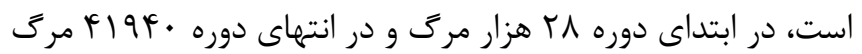

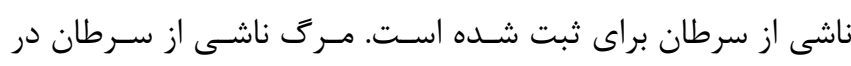

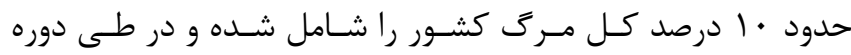
افزايشى بوده است (جدول شماره ()).

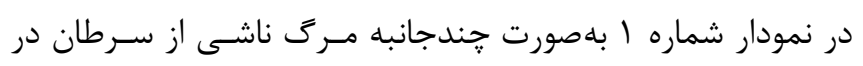

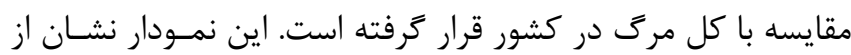

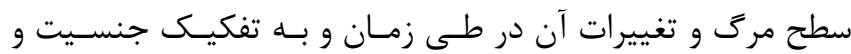

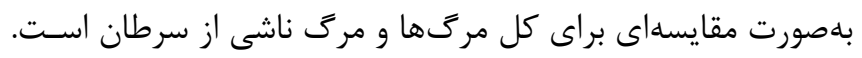

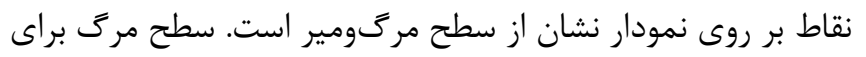

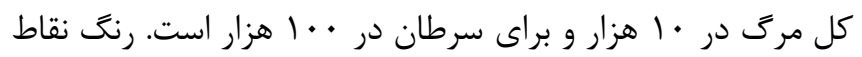

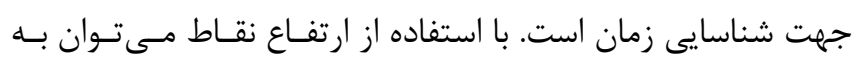

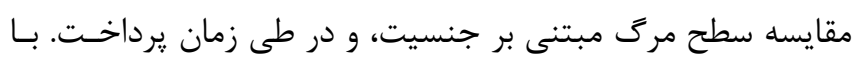

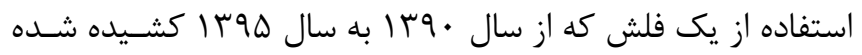

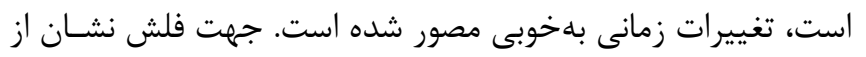

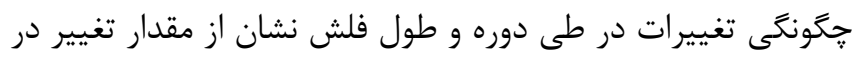

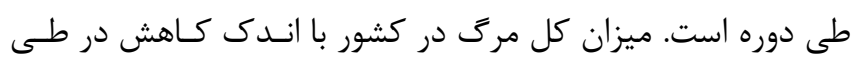

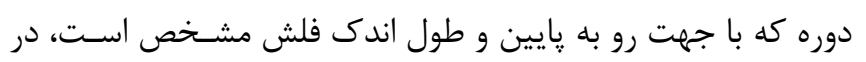

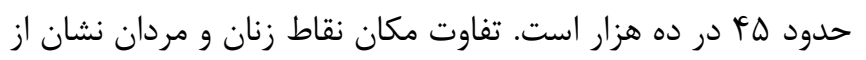

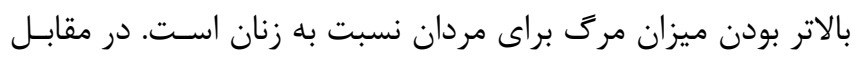

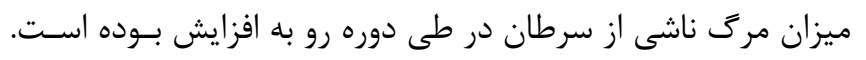

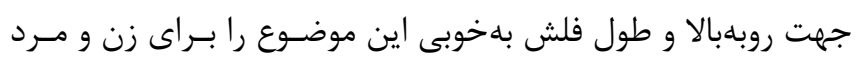

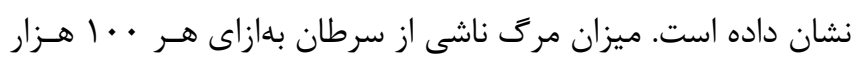

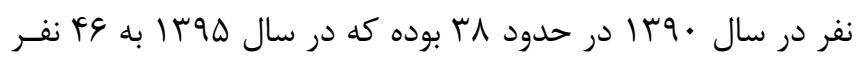

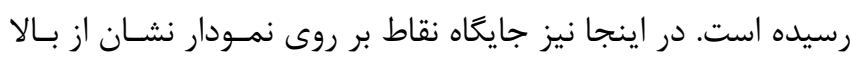

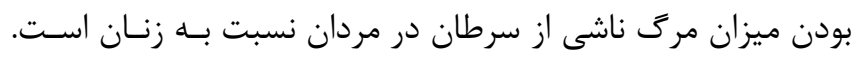

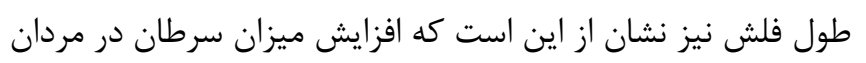

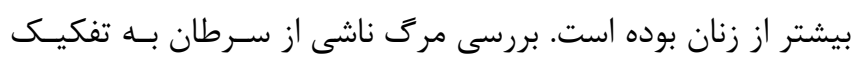

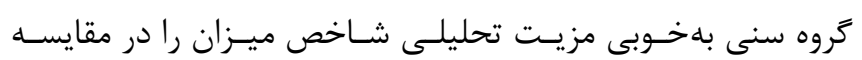

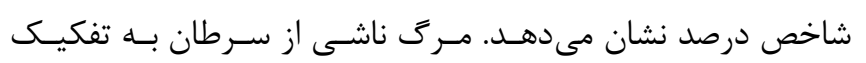

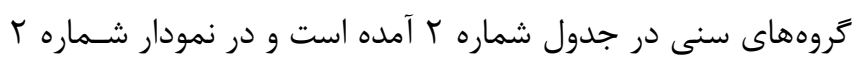

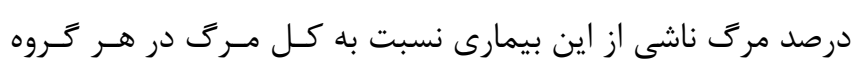

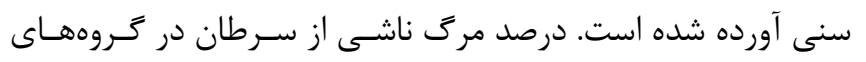

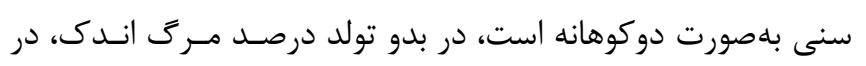

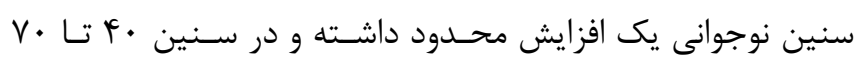

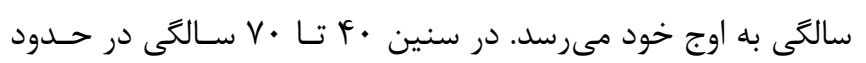

طبقهبندى بينالمللى بار بيمارىها (ICD10) استخراج شده اسـت.

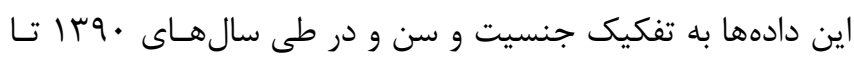

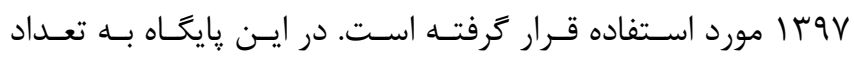

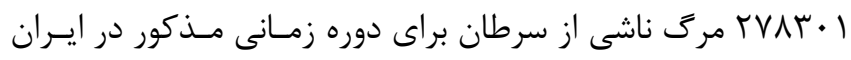

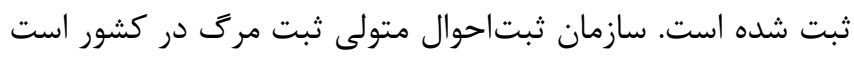

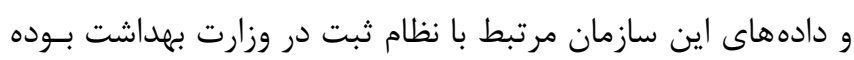

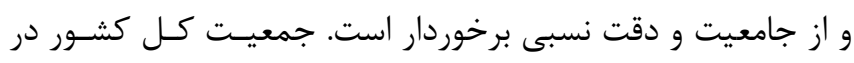

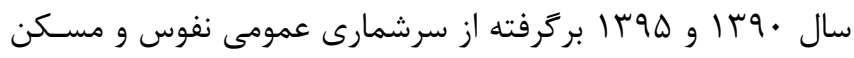

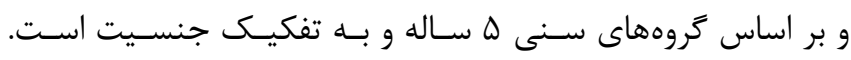

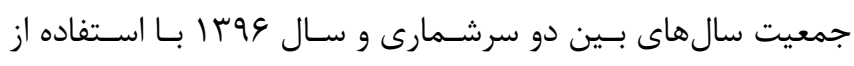

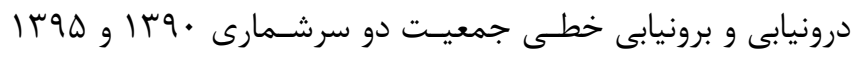

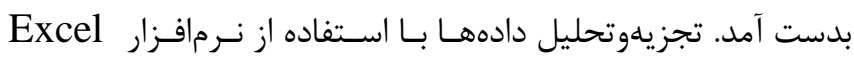

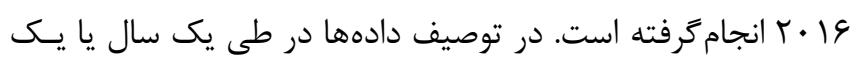

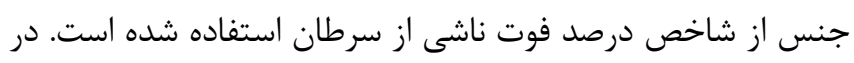

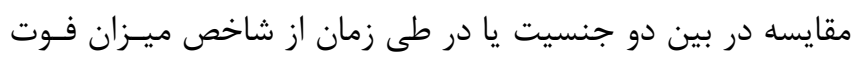

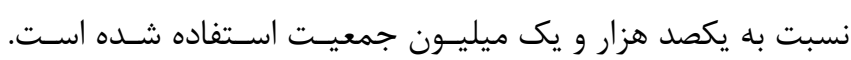

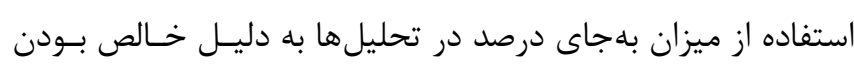

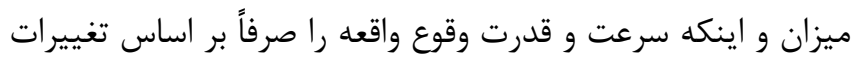

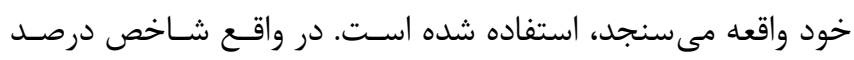

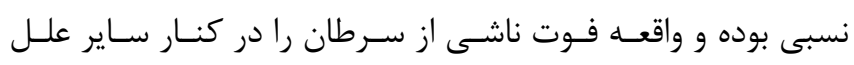

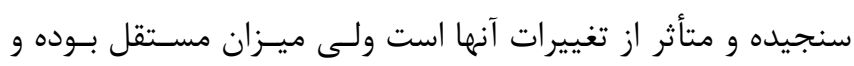

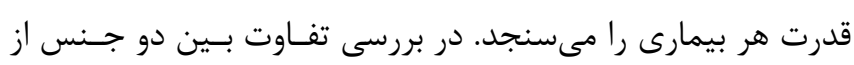

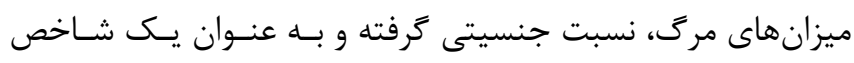

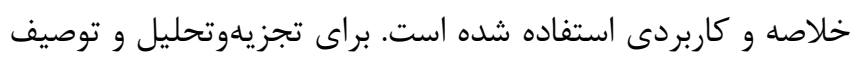

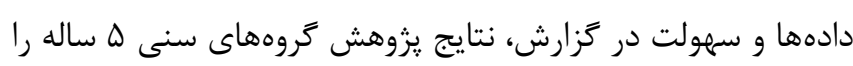

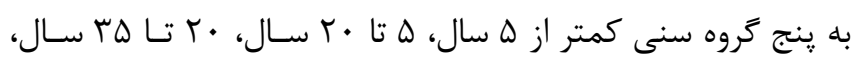
هץ تا •D سال و بالاى • • سال تقليل داده شده است.

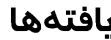

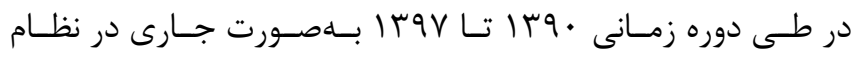

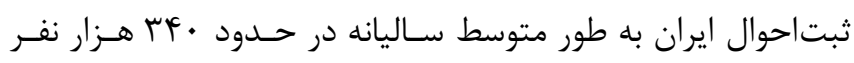

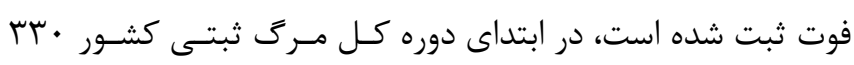

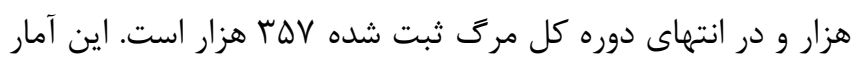

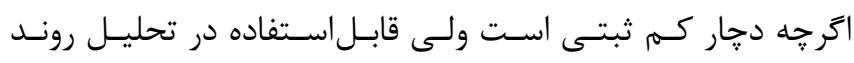

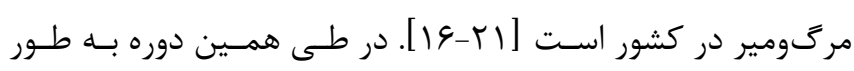

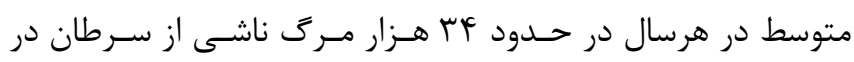


با استفاده از شاخص نسبت جنسى انجام شده است. اين شاخص در

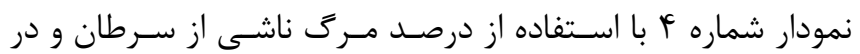
نمودار شماره ه با استفاده از ميزان مرگ محاسبه شده است.

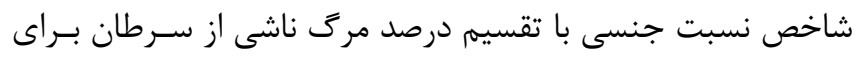

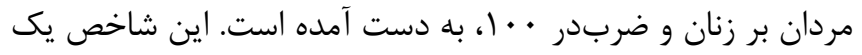

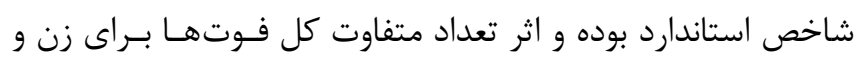
مرد را حذف نموده است و در معنى آمارى نشان از اين است كه آر إن

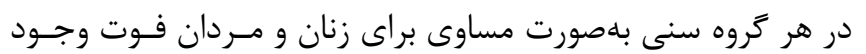

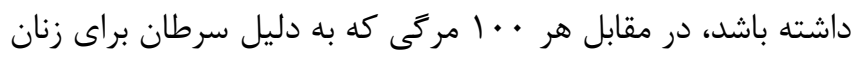

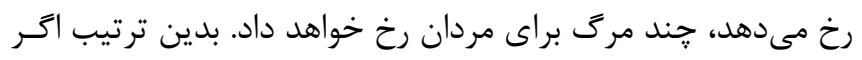
مقدار شاخص برابر . . أشود يعنى سهم فوت ناشى از سرطان براى

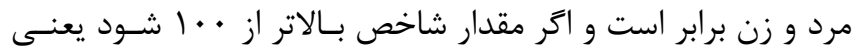

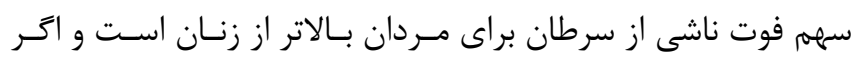

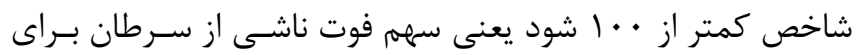

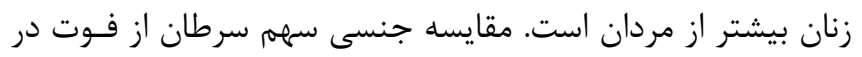

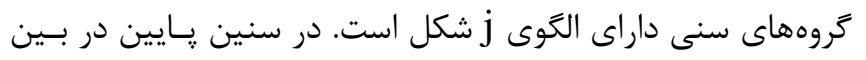

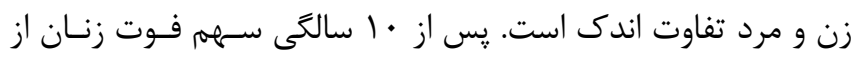

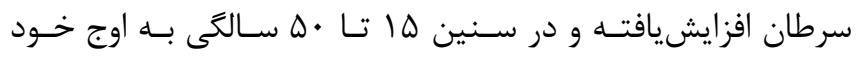

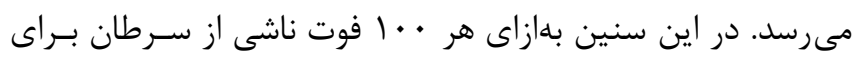

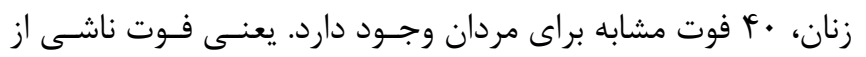

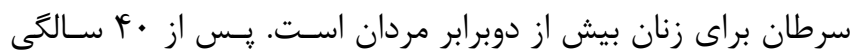

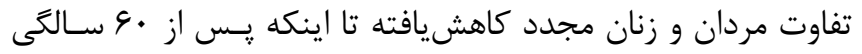

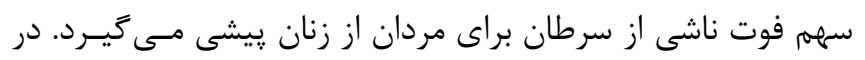

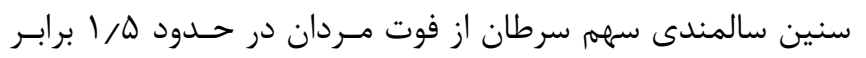

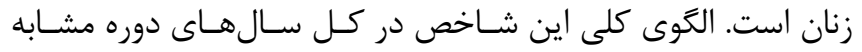

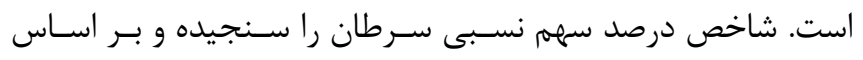
شاخص ميزان قدرت مطلق سرطان در مرك بر اساس سـن در بــين

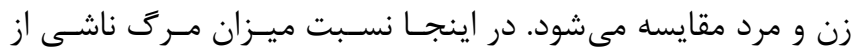

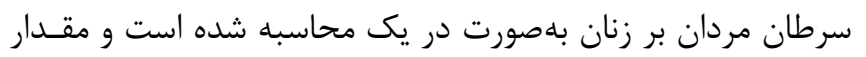

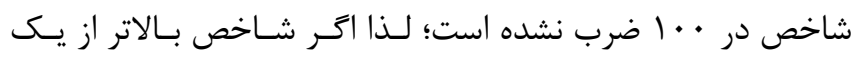
باشد نشان از بالا بودن مرگ مردان نسبت به زنان و برعكس اسـت.

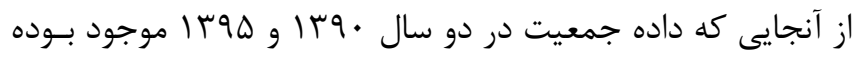

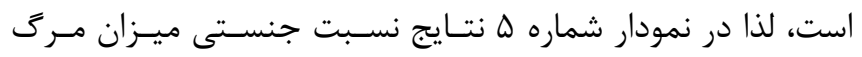

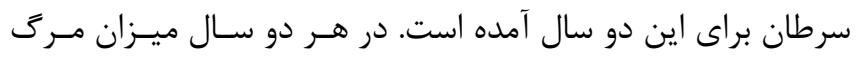

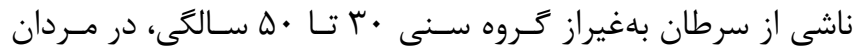

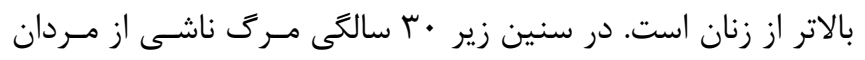

د درصد مرگ ناشى از سرطان است. مقايسه درصد مرگ ناشسى از IV

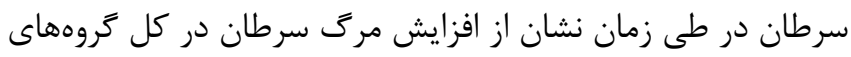

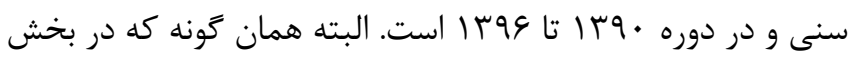
روش ذكر شد شاخص درصد نسبى است و متـأثر از تغييـرات سـاير

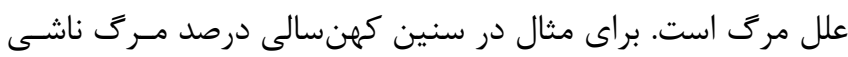

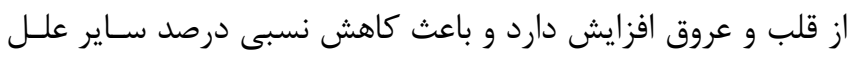

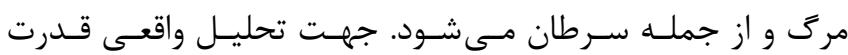
مرى آورى سرطان در طى سـن در نمـودار شـماره ب ميـزان مـرى ناشى از سرطان محاسبه و به بهصورت لكاريتم به تصوير آمده است. (استفاده از لعاريتم به لحاظ تكنيكال تمايزات بين مقادير كوجـك و بزرى را بهتر نشان مى دهد). همان گَونه كه در اين نمودار مشـخص

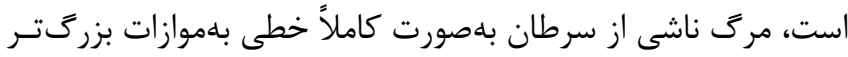

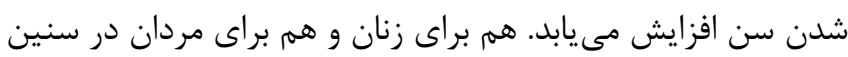
بالاتر ميزان مرى ناشى از سرطان افزايش يافته است.

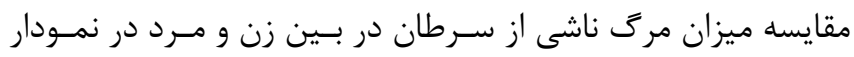

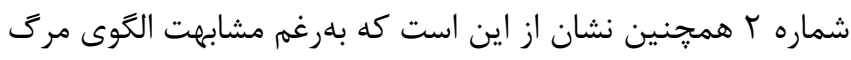

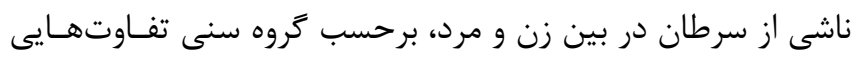
در بين اين دو وجود دارد. همان گونه كه در مبحث قبلى مشـاهده

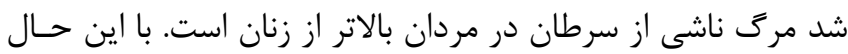

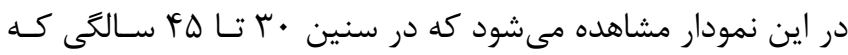
مربوط به سنين باردارى زنان است، ميزان مرگ ناشى از سرطان در بين زنان بالاتر از مردان است. ميانگين سنى مرگ ناشى از سـرطان

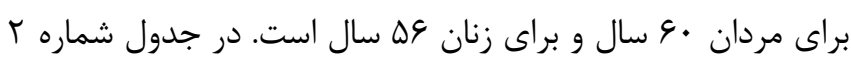

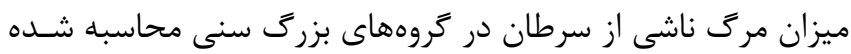

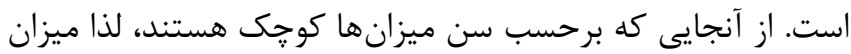

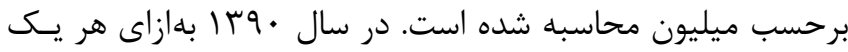

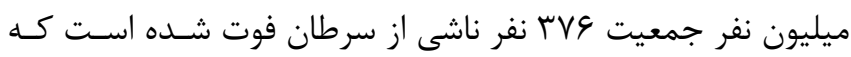

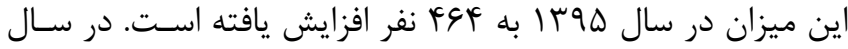

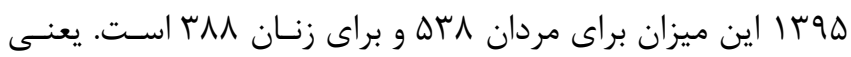

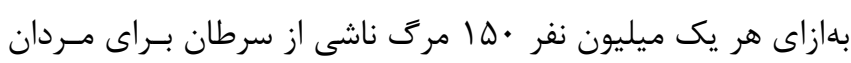

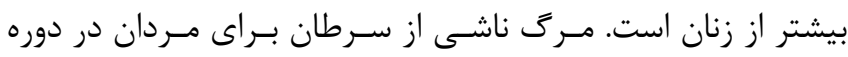

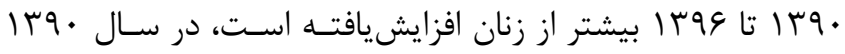
تفاوت ميزان مرى زن و مرد 11 ا نفر در ميليون بوده است. ميـزان

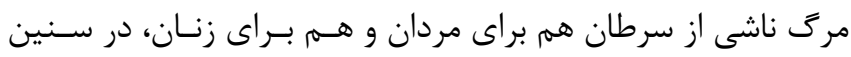

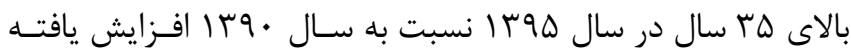

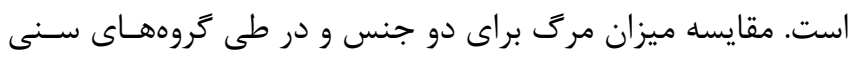


نشريه يروهشكده علوم بهداشتى جهادانشعاهى

هزار آن مربـوط بــه سـرطان بـوده اسـت. مجمــوع سـالهــاى عمـر

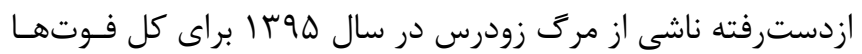

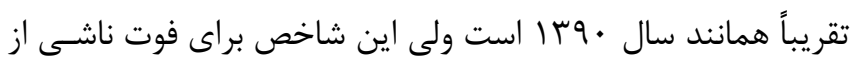

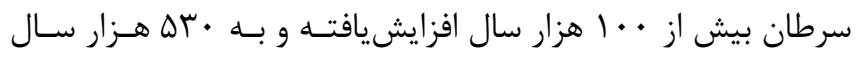

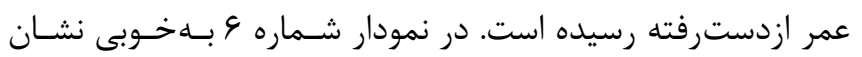
داده شده است كه عامل افزايش سالهاى عمر ازدسترفته ناشسى از اردار

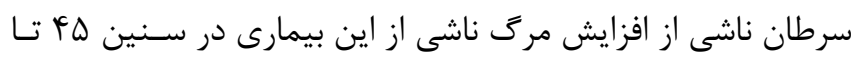

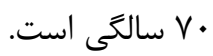

در نمودار شماره V تفـاوت سـالهـاى عمـر ازدسـترفتـهـ ناشـى از

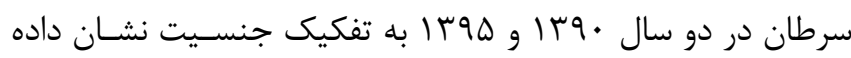
شده است. افزايش مرگ ناشى از سرطان براى هر دو جنس مربـوط

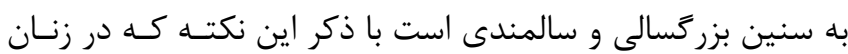
ميانسال تا حدودى بيشتر از مردان و در مـردان سـالخورده مقــدار

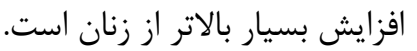

حدوداً r ا برابر زنان است و در سنين بالاى • ه سالخى اين نسـبت

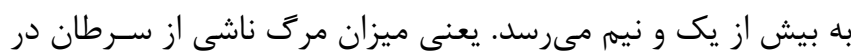

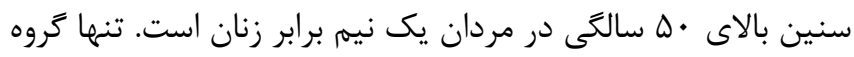

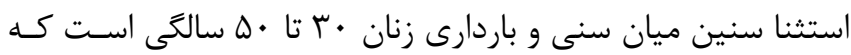
در آن ميزان سرطان براى زنان بيشتر از مردان است و نسبت بان به زئنير

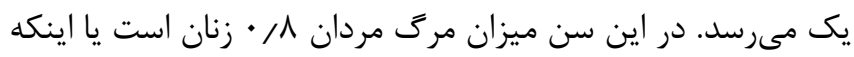

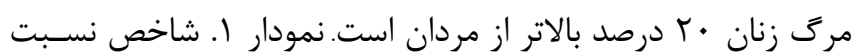

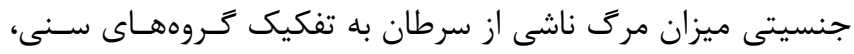

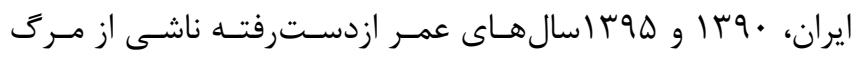

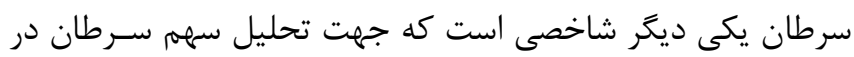

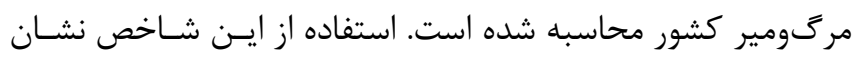

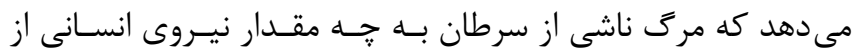

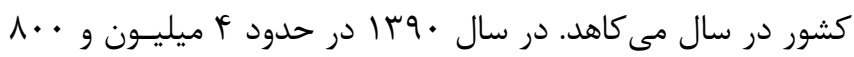

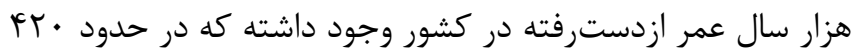

\begin{tabular}{|c|c|c|c|c|c|c|c|c|c|}
\hline 11798 & 1499 & 11790 & 1794 & 1494 & 1794 & 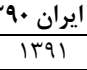 & 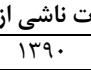 & سال سد & جدول ا: ت \\
\hline$\wedge 1, \wedge$ & $\Lambda \cdot, \wedge \Lambda$ & V१,१r & 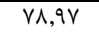 & $\mathrm{VA}$ & $V V$ & VE, I. & $V \Delta, 1 f$ & & كل جمعيت (به ميليون) \\
\hline 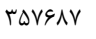 & TVEAFq & $r F \Lambda . F q$ & rrugft & (1וाז & TrETr & rT. 991 & $r r \cdot 9 v r$ & جمع & كل فل فوت \\
\hline$r \cdot r 190$ & $r 1.91 r$ & 191910 & IqFAFT & $|194| 1$ & I9T०9T & $|\Lambda F \cdot \Delta|$ & 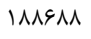 & 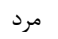 & بت شده \\
\hline IDFFAT & 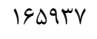 & Ifqgाr & $|f f| \ldots$ & $|f| \wedge r q$ & $1 f r q \cdot \Lambda$ & 1 1r99.. & Ifresq & 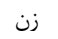 & \\
\hline fifq. & rqFVD & $r v \cdot \Lambda \cdot$ & rov^l & reqIV & rrtql & 「८१५। & TArTS & جمع & وت ناشى از سرطانها ر \\
\hline$r F \cdot r F$ & rTAIT & rIVqV & $\langle 1 \cdot \Delta \varphi$ & r.rवr & 11994 & $|V \cdot \Delta|$ & IEFV & 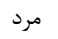 & ومورها \\
\hline IVFAG & 19994 & $10 r q$. & IFVTD & IFQT. & $I M F \cdot \Delta$ & $119 \cdot 4$ & $11 \vee \wedge 9$ & 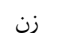 & \\
\hline $11 / 9$ & $1 \cdot / \Delta$ & $1 \cdot / \mathrm{V}$ & $1 \cdot 19$ & $1 \cdot / 0$ & $9 / 9$ & $q / \cdot$ & $\Lambda / \Delta$ & جمع & مرصد فوت سرطان و تومور \\
\hline $11 / 1$ & $1 \cdot / 1$ & $11 /$. & $1 \cdot / 1$ & $1 \cdot / 1$ & $9 / 9$ & $q / r$ & $\Lambda / \vee$ & مرد & كل فوت \\
\hline $11 \pi$ & $1 \%$ & $1 \cdot \pi$ & $1 \cdot \pi$ & $1 \cdot \pi$ & $q, r$ & $\Lambda / \vee$ & $\Lambda, r$ & زن & \\
\hline
\end{tabular}

\begin{tabular}{|c|c|c|c|c|c|c|c|}
\hline \multirow{2}{*}{ Iras } & \multirow[b]{2}{*}{$1 \pi 90$} & \multirow[b]{2}{*}{ Iraf } & & \multicolumn{4}{|c|}{ 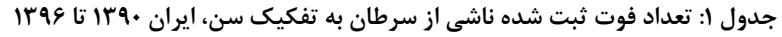 } \\
\hline & & & irar & irar & $|1 r 9|$ & $1 \% q$. & سن \\
\hline \&) & 49 & $\Delta T$ & $F \Delta$ & FT & $F \Delta$ & fr & $\cdot$ \\
\hline IVA & $1 \Delta \Delta$ & 191 & 149 & 19. & 110 & 109 & $f-1$ \\
\hline rII & lof & $14 q$ & Ist & IST & If. & IrV & $9-\Delta$ \\
\hline 199 & $19 \mathrm{~V}$ & $1 \Delta \Delta$ & $\mid \Delta F$ & 1149 & 149 & If. & $\mid f-1$. \\
\hline rוr & $r \cdot 1$ & $r 1$. & TTV & TrA & rrq & r94 & $19-10$ \\
\hline$r \cdot q$ & rVA & r৭D & ref & TSV & reᄉ & $|F|$ & $r F-r$. \\
\hline 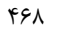 & YAD & $\Delta \cdot 1$ & DrG & QIT & Qr. & $\Delta V r$ & $r q-r \Delta$ \\
\hline NFr & VAr & 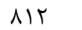 & V99 & 999 & st4 & GVG & $\mu r-r$. \\
\hline $11 \ldots$ & $1 \cdot F r$ & $1 \cdot r \cdot$ & $q \Delta F$ & $\Lambda \Delta r$ & VAT & $\checkmark V \Delta$ & ט \\
\hline $14 \cdot 9$ & $|r \Delta|$ & $1 \pi 9 \Delta$ & $|M F|$ & ITFT & $11 \cdot r$ & 1.99 & $f F-f$. \\
\hline$r \cdot 19$ & $19 \mathrm{VT}$ & INGY & IVTF & IEr & $\mid \Delta F \Delta$ & If $F$ & $q q-i \Delta$ \\
\hline TENA & rब१८ & TATD & rQSD & TFF & rITr & rIDS & $\Delta \mathcal{F}-\Delta$. \\
\hline ए१८ & rVrq & THEl & $r F \Delta \Lambda$ & عسוس & rVTE & TEVT & $\Delta q-\Delta \Delta$ \\
\hline 4019 & FTVI & $r \cdot I r$ & ryeq & & rqAr & rVIT & sq-G. \\
\hline fQAST & fiqu & rarv & rNDF & TFVT & $r \cdot I V$ & rVAS & $99-90$ \\
\hline FrAV & $f \mid \Delta$. & rqve & rArq & TGTF & mpqq & TFII & $V F-V$. \\
\hline IrIV. & $11 \Delta \Leftrightarrow \Delta$ & IITAT & $11 . r 4$ & $1 \cdot T M \Lambda$ & $91 \Delta \Delta$ & AVYI & $+V \Delta$ \\
\hline rqfrk & $r v \cdot v \cdot$ & $r \Delta V V r$ & rF^qץ & rrv. & rیq।r & TATKG & جمع \\
\hline
\end{tabular}

مآخذ: استخراج از دادههاى سازمان ثبتاحوال ايران 


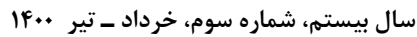

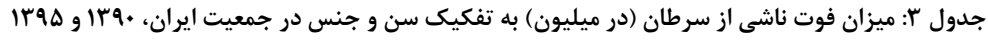

\begin{tabular}{|c|c|c|c|c|c|c|c|c|c|c|}
\hline \multicolumn{3}{|c|}{ ميزان (در ميليون) } & \multicolumn{3}{|c|}{ فوت ناشى از سرطان } & \multicolumn{3}{|c|}{ جمعيت كل } & \multirow[b]{2}{*}{ كروه سنى } & \multirow[b]{2}{*}{ 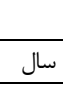 } \\
\hline زن - ان & مرد & جمع & زن & 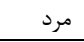 & جمع & زن & مرد & 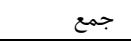 & & \\
\hline rی & $\Delta \Gamma \Lambda$ & kq4 & IQTAY & riva. & $r V .9 \%$ & TQFTVATA & F.FqAFFr & V৭१५G Y. & كل & \\
\hline rq & rr & rq & 19 & 110 & $r \cdot r$ & referty & rqpqu.. & $v \cdot q r \cdot .4$ & كمتر از هـ & \\
\hline 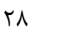 & re & $r \cdot$ & rtr & TAD & $\Delta T r$ & $\Lambda \Delta V \Delta D I V$ & 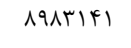 & $\mid \vee \Delta \triangle \wedge \wedge \triangle \Delta \Lambda$ & $r \cdot-\Delta$ & \\
\hline v. & sV & $q V$ & V\&l & $\checkmark \wedge \Delta$ & IDFS & $\| F V F \cdot \Delta V$ & IIVT·A & rTlqfard & $r \Delta-r$. & $114 \Delta$ \\
\hline rVr & trt & TFV & rma & 199. & frqT & $\wedge \Delta F \wedge r \cdot \Lambda$ & $M A F \cdot V T$. & $\mid V r \wedge q \cdot r \Lambda$ & $\Delta \cdot-r_{\Delta}$ & \\
\hline 19.9 & rQDS & $r \cdot v G$ & 1114. & 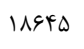 & $r . r q \Lambda$ & VTAGVYFT & vr.rqाr & $1499.9 \Delta 0$ & له به بالا & \\
\hline ris & rta & rVe & $\| \gamma \wedge \Delta$ & $\mid 94 \mathrm{VI}$ & TAKTS & rVtef... & rVq. ఎ૬૬q & VDIFQ९99 & كل & \\
\hline r & re & זr & 99 & $1 \cdot v$ & $r \cdot r$ & $r \cdot Y \cdot Y Y_{I}$ & rIqTाII & GTHTAQT & كمتر از هـ & \\
\hline rq & rt & $r \cdot$ & $r \Delta \Delta$ & TMA & DFT & $\Lambda \Lambda \cdot 1 \Lambda \Lambda \Delta$ & 9ITFYAF & IVArGTG9 & $r \cdot-\Delta$ & 9. \\
\hline$\wedge$. & 91 & $v \cdot$ & $9 \Delta \Delta$ & ve. & 199. & $1194 v \cdot r \wedge$ & $M r \cdot V r \cdot r V$ & $T F \cdot \Delta q \cdot V \Delta$ & $r \Delta-r$. & 119. \\
\hline rat & $r \cdot \Lambda$ & $r r$. & $11 \cdot 9$ & lata & rrr. & VIFFTaA & VКятqD. & $I F \Delta \cdot A T F A$ & $\Delta \cdot-r_{\Delta}$ & \\
\hline IrAv & TTOV & 1119 & 19VT & $\mid r \Lambda \cdot 1$ & rrFe. & GTATVYY & 9114499 & ITMGVT.r & . ه به بالا & \\
\hline
\end{tabular}

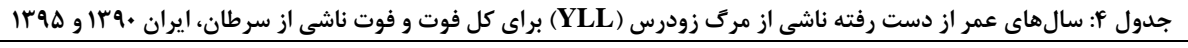

\begin{tabular}{|c|c|c|c|c|c|c|c|c|c|c|c|c|}
\hline \multicolumn{6}{|c|}{$1 \% 9 \Delta$} & \multicolumn{6}{|c|}{$1 \% q}$. & \multirow{3}{*}{ كروه سنى } \\
\hline \multicolumn{3}{|c|}{ سرطانها و تومورها } & \multicolumn{3}{|c|}{ كل فوت } & \multicolumn{3}{|c|}{ سرطانها و تومورها } & \multicolumn{3}{|c|}{ كل فوت } & \\
\hline زن & مرد & جمع & زن & مرد & جمع & زن & مرد & جمع & زن & مرد & جمع & \\
\hline AYF & $49 \mathrm{~V}$ & $\mid 4 q 1$ & 110191 & $|r \Delta \Lambda \Lambda|$ & $r \Delta I \cdot V r$ & $\Delta \Lambda$. & $V \Delta \Lambda$ & ITrA & $\mid$ IFTा| & IVAGF. & $T T \backslash \wedge \Delta$. & . \\
\hline$|111|$ & rVqq & $\forall \notin 1$. & $\Delta \cdot \Delta r$ & 9.19 & $11 \cdots V T$ & פצre & TFEN & $\psi_{\lambda} \cdot \hat{r}$ & GITE & $8 r \cdot \Delta q$ & 11 frad & $r-1$ \\
\hline TITK & $r F \Delta \Lambda$ & $f \Delta \Lambda$. & T. TF. & TVQA. & SVAT. & rTII & 1149 & $r \cdot v V$ & $r q \cdot V \Delta$ & rvq. & 99911 & $9-\Delta$ \\
\hline TTAS & rG. F & Fへ૬. & rVt.. & TATED & \&Y990 & $I V \wedge \Lambda$ & TTAD & $r \cdot v r$ & тя१६. & $r \Delta \Delta \Lambda r$ & STDFT & $\mid f-1$ \\
\hline TAFV & MIDq & $\Delta V \cdot \Delta$ & एद्व & NGFIF & 1 ITK. 9 & metr & 4111 & $V \Delta \Delta I$ & 49991 & $1 \cdot \vee 9 \wedge 9$ & IDFGDS & $19-10$ \\
\hline TrI. & frov & VGAV & FFole & ITIVEF & ISGTA. & GQTI & $\Delta G \wedge \varphi$ & ITTIV & AAVTD & IVGqVF & rצD999 & $r F-r$. \\
\hline GIVT & GAT. & Ir995 & घヘr.r & IFroAv & rIIAq. & qVVr & $\Delta V \cdot \Delta$ & IDFVA & 1.9151 & 189.99 & rMAIqT & rQ-ro \\
\hline $1 \cdot 111$ & 9YAI & $r .199$ & VVEFA & IDTVYF & rtert & qrVV & $\Lambda \cdot \wedge \varphi$ & IVEAT & $V V \cdot r r$ & $|\Delta \Lambda| F$. & rTDIET & 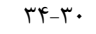 \\
\hline IFEAT & $1.9 \Delta F$ & TASTA & 99Уध9 & $|F \Delta r \cdot|$ & $r I D \cdot r$. & 1.019 & $\Lambda \Delta \varphi$. & $19 \cdot 19$ & DGGAT & ITYFTV & 119119 & rq-ro \\
\hline IVFVE & ITTE. & rqvrs & $G \Delta V \Delta F$ & IrFroq & $r \cdot .11 r$ & IF्9 & 1.994 & $r \Delta \& \Delta q$ & $9 \Delta \cdot+9$ & Irgark & $r \cdot \mid \Delta \wedge \Delta$ & $f f-f$. \\
\hline TrIAT & $r \cdot T \Delta I$ & FroTF & $\Lambda \cdot \Delta \Leftrightarrow \varphi$ & IDFVQA & rTargF & ISV9V & IfEVD & rifVT & VVद৭9 & IFVFAT & rTDIVA & $F q-F \Delta$ \\
\hline$r \Delta \cdot I V$ & ratrq & DITQG & qGFDF & INIATV & TVATAI & $r \cdot I V r$ & TrFFE & FTETK & 1. rVGF & MffFr & rAVI91 & $\Delta F-\Delta$. \\
\hline rq১१ & reTFF & 909भद & $|r \wedge \Delta T|$ & TrISID & ro. $1{ }^{\prime}$ & TIMTK & roqFT & FVTEY & ITTAIS & IAVETA & TI. FFS & $\Delta q-\Delta \Delta$ \\
\hline rqוג & rGVDS & яด१९q & IOKGVV & TIETIT & एव9 & 11994 & גי... & figrr & Ir.VAr & $19 \cdot 194$ & rq19VG & $s f-s$. \\
\hline rrofq & $r .999$ & $\Delta F \Delta F \Lambda$ & $|\Delta \Delta| \cdot \varphi$ & IVDVG. & 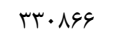 & $10 \cdot 49$ & TIIVT & TETTI & $|r p q|$. & IFVr人q & TATI9A & $99-90$ \\
\hline MITS & rDSAF & rrs. $q$ & Ifqr.r & logqVT & $r .9 I V \Delta$ & $10 \cdot 1 f$ & $r l \ldots r$ & re.IV & $|\Delta \Delta F V|$ & I & פזדשזr & $V f-v$. \\
\hline rglar & DAVIT & 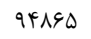 & GqVFVD & GTIDTH & Iтr人११人 & $r V \cdot V \Lambda$ & fFeft & VIDT. & $9 r \cdot 109$ & $\Delta V \cdot r \Lambda l$ & $\| r \cdots \Delta F$. & $+V \Delta$ \\
\hline$r F \Delta \Lambda \Delta \Lambda$ & $r q \cdot \varepsilon \cdot \Delta$ & DrGYG & r. rETr. & rVqIधDT & FArVqTr & 19DGFT & DTINA & FIAMTA & T. FATOT & TVVTT.V & $F \wedge r \cdot \Delta \Delta q$ & جمع \\
\hline
\end{tabular}

- irad o ira.

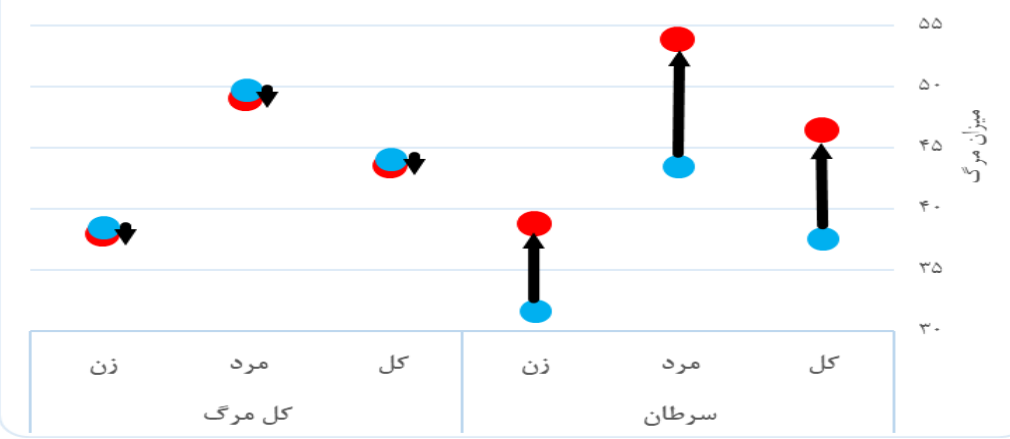

نمودار ז: ميزان مرى كل (در •اهزار) و ناشى از سرطان (در •.ا هزار) به تفكيك جنس، ايران •وسا و هqسا 


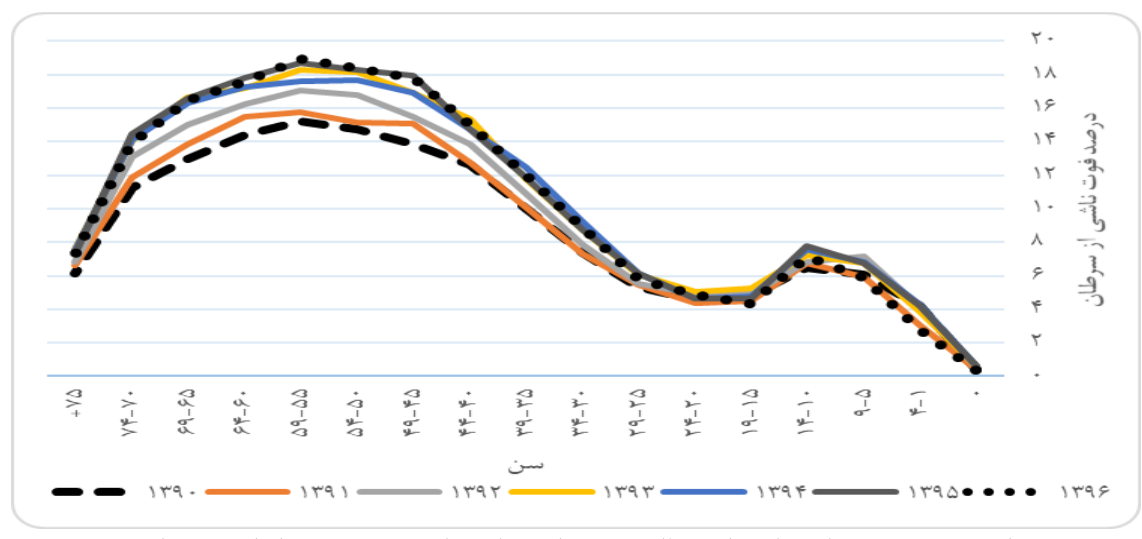

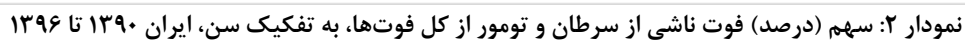

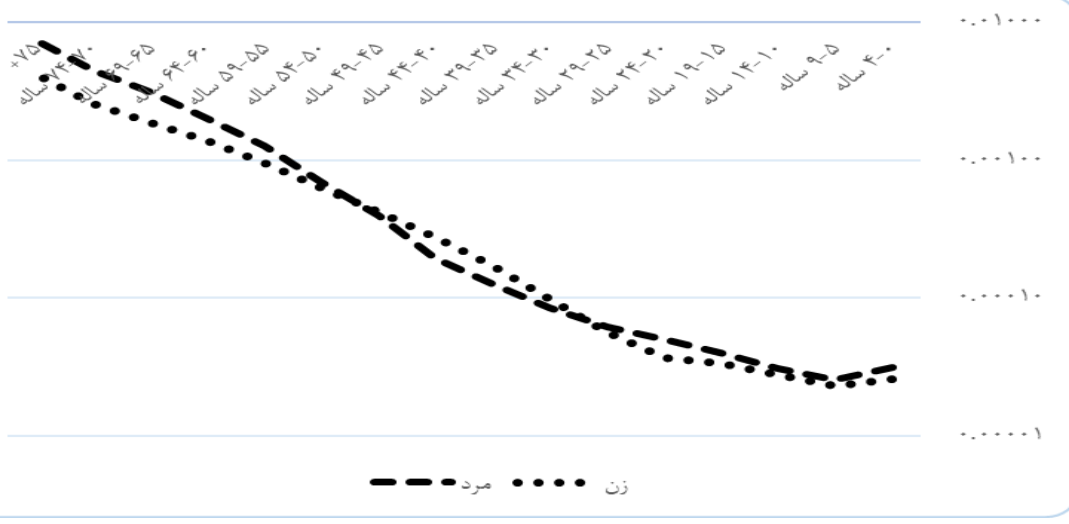

نمودار بّ: لكَاريتم ميزان مركى ناشى از سرطان به تفكيك سن و جنس، ايران هوس|

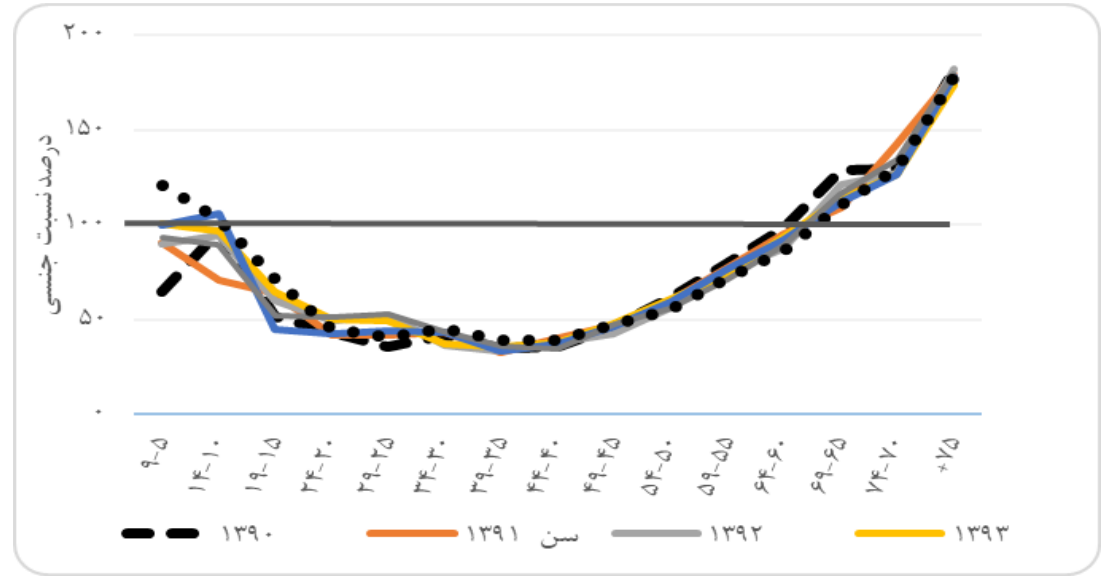

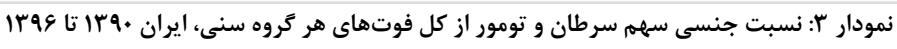


سال بيستم، شماره سوم، خرداد ـ تير ..1F

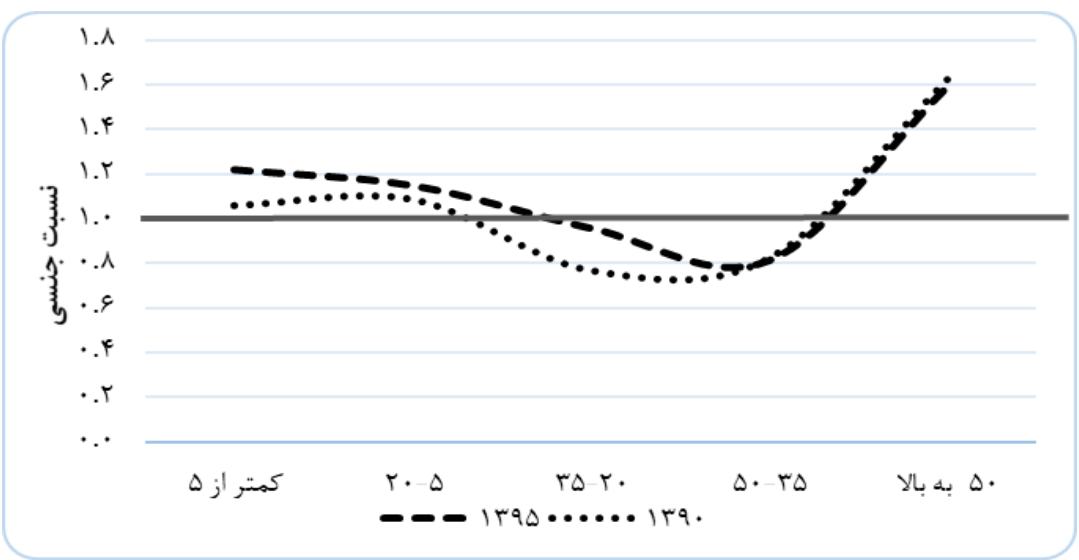

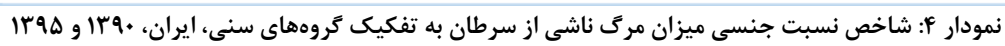

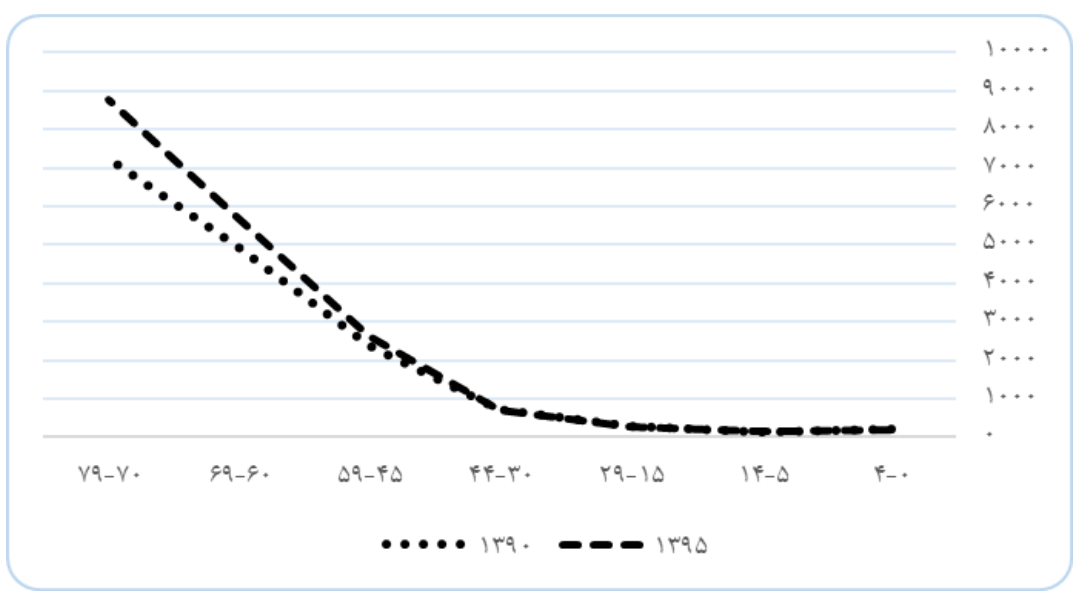

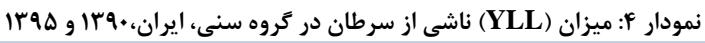

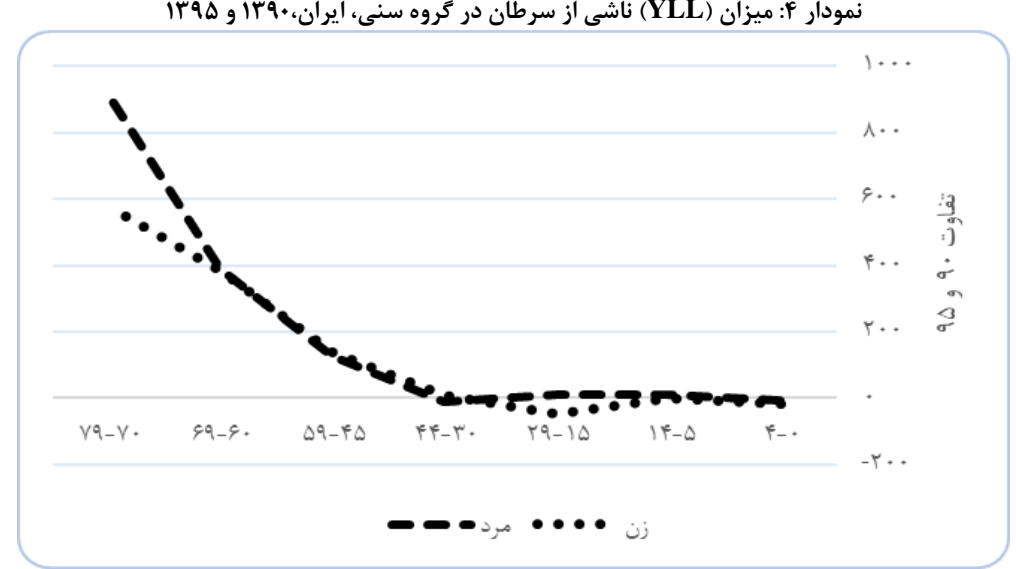

نمودار ه:تفاوت ميزان (YLL) ناشى از سرطان در دو سال •qجا تا هو با به تفكيك جنسيت، ايران.

تولد اندك، در سنين جوانى يك افزايش محدود داشته و در سـنين

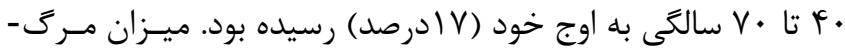
ومير به دليل بيمارى سرطان بر اساس جنسيت، در گرووهاى سـنى إنى

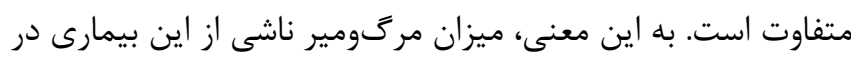

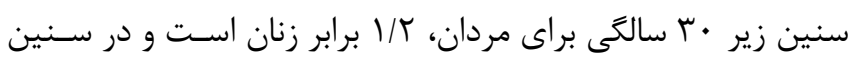

بحث و نتيجه گيرى

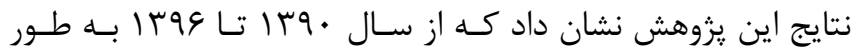
متوسط سالانه حدود سج هزار نفر به دليل سـرطان در ايسران فـوت كرده اند. در طول دورهى مطالعه ميزان مركىومير ناشىى از سـرطان

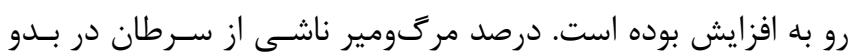


كه ميزان كل مركَومير ناشى از سرطان دستخاه گوارشى در مـردان

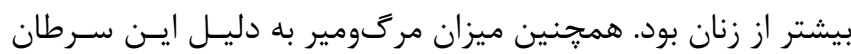

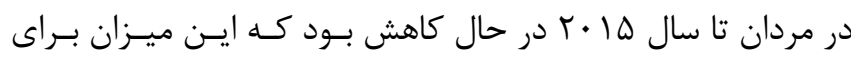

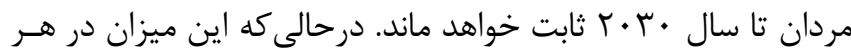

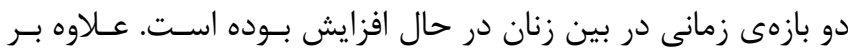

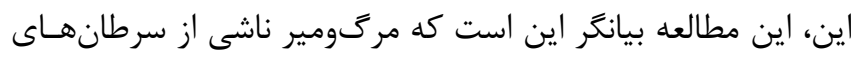

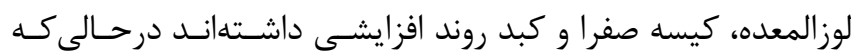

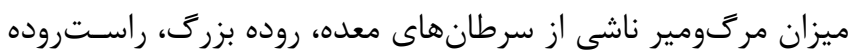

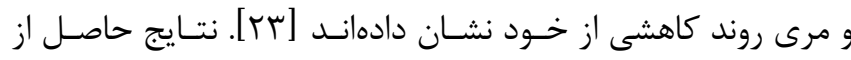

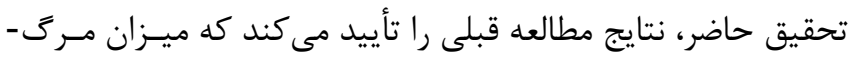

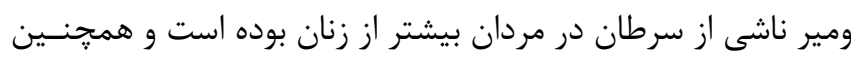

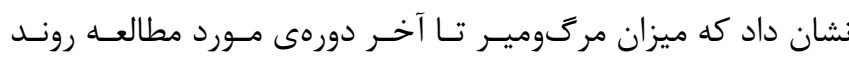

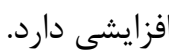
مطالعه يان و همكـاران در كاليفرنيـا بيـانكر ايـن اسـت كـه شـيوع

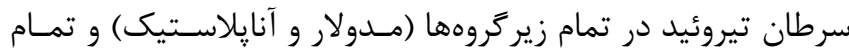

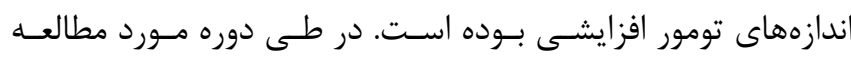

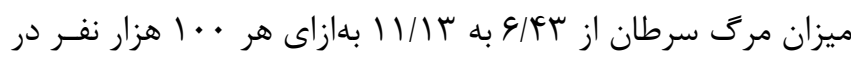

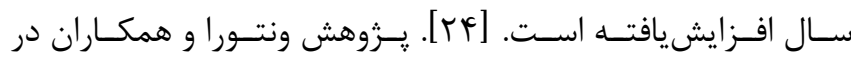

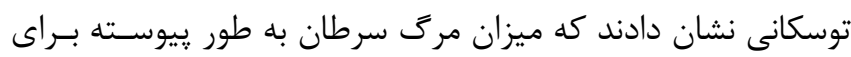

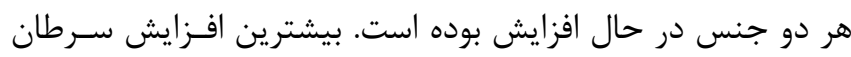

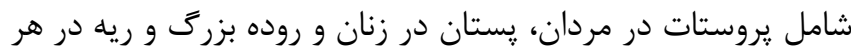

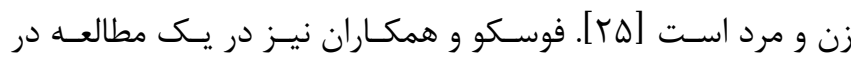

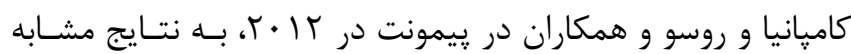

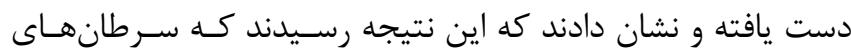

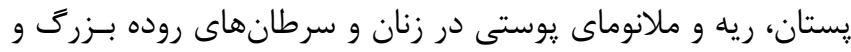

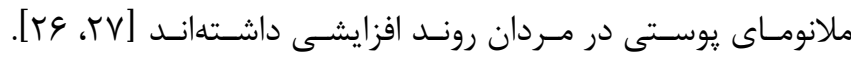

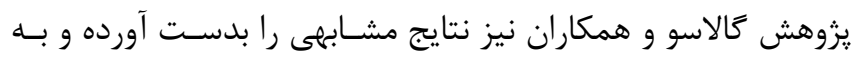

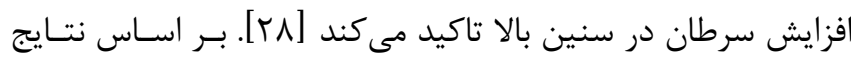

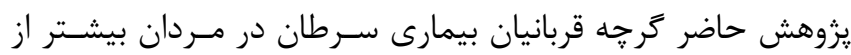

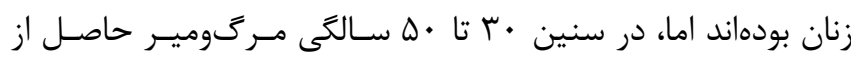

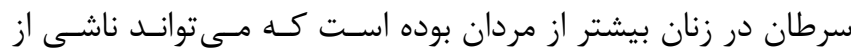

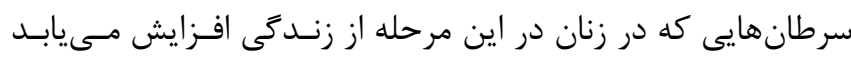

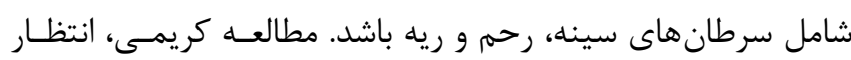

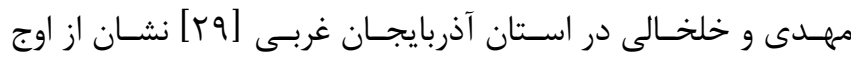

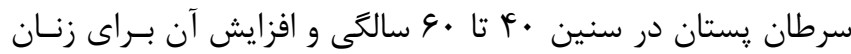

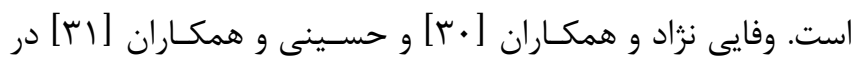

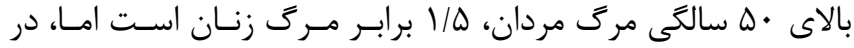

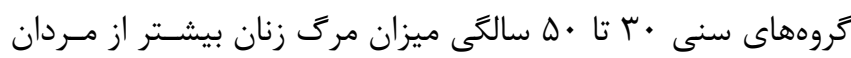

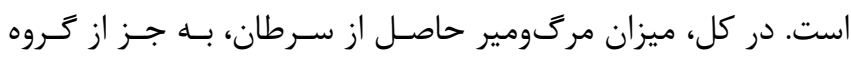

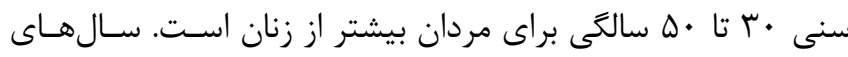

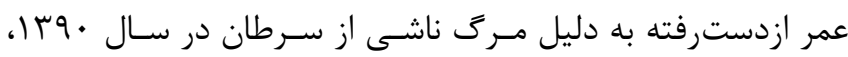

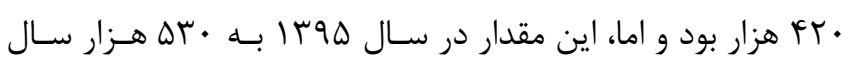

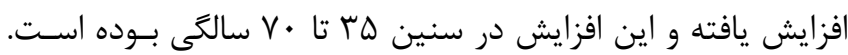

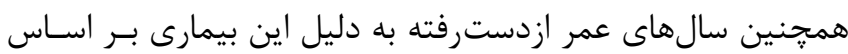

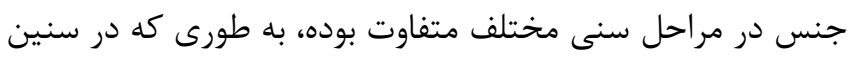

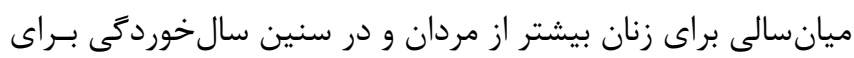

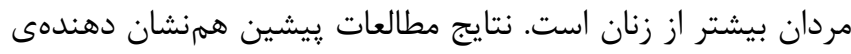

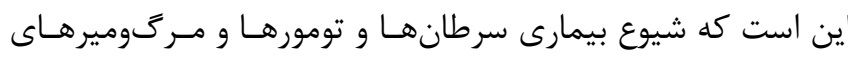

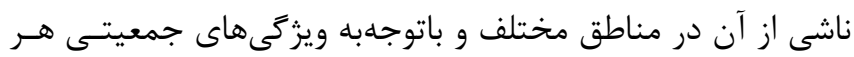
منطقه و زمان متفاوت است. تعداد از مطالعات كه در ايـن يـروهش

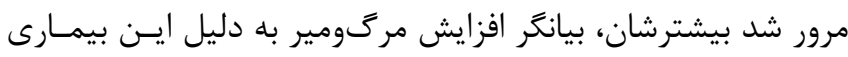
در سال هاى اخير بوده است.

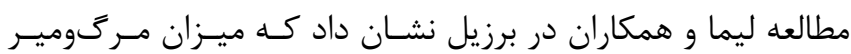

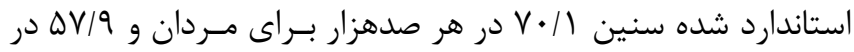

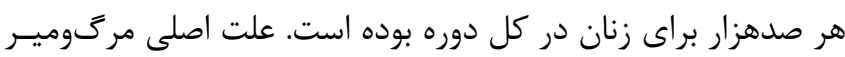

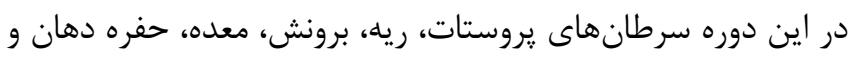

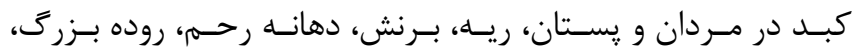

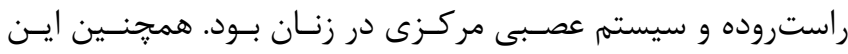
تحقيق نشان مىدهد كه منحنى هاى مركَ

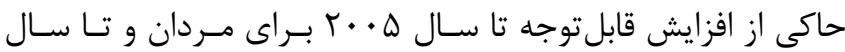

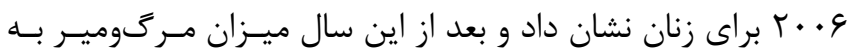

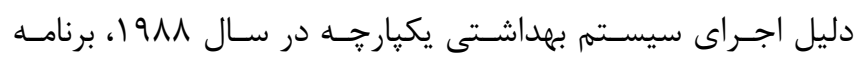
بهداشت خانواده، تشويق دولت براى متخصصان بهداشت بـراى كـار

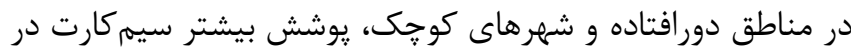

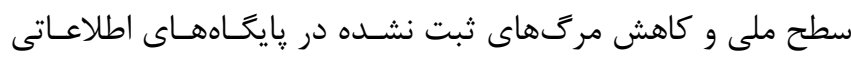

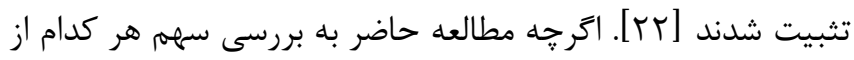

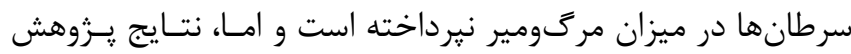

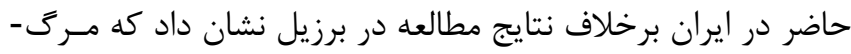

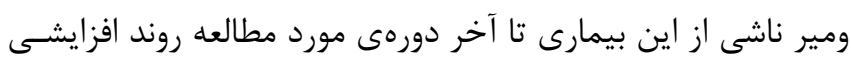

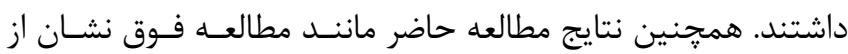

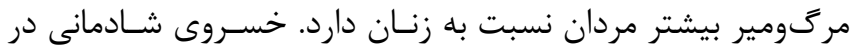

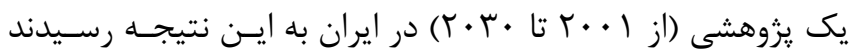


كشورهاى ديخر انجام شده است، مى توان نتيجـهـ كَرفـت كـهـ مـرى

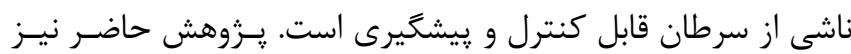
بيشنهاد مى كند كه براى كاهش شيوع و ميزان مركَوميــر ناشسى از

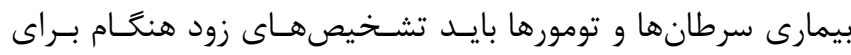

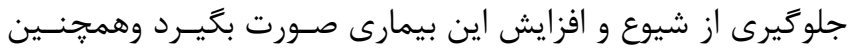

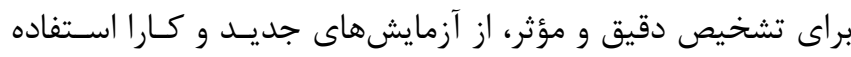

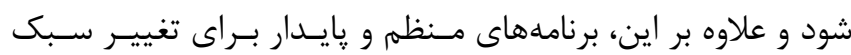

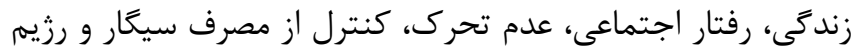

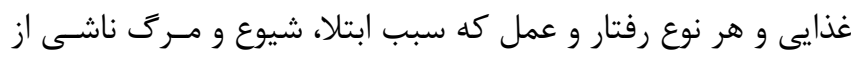
سرطانها مىشود برنامهريزى و فرهنَّسازى شـود. مـرى ناشـى از

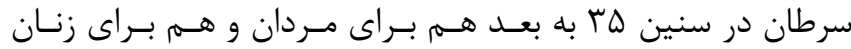

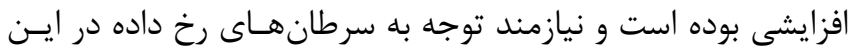

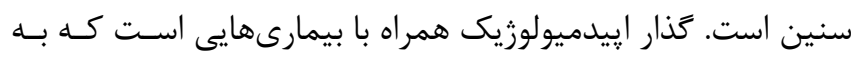

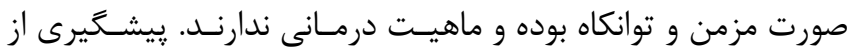

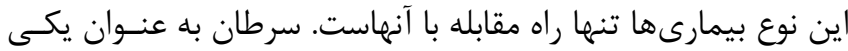

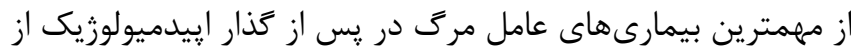

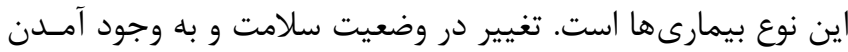

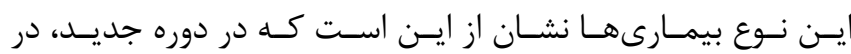

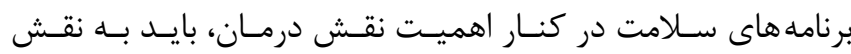

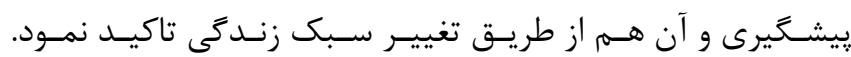

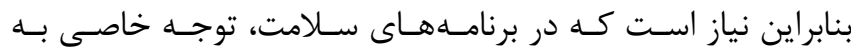

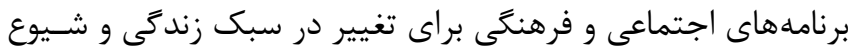
رفتارهاى سلامت محور در افراد اقدام نمود.

\section{سهم نويسند كَان}

محمد تر كاشوند مرادآبادى: طراحى و نعارش مقاله، تفسير و تحليل دادهها، طبقه بندى و ارزيابى دادهها، جمعبندى نداني واحد سروش: تحليل داده، تنظـيم جــداول و ترسـيم نمـودار، مـرور بيشنه مطالعاتى زهرا تر كاشوند: ارزيابى دادها، تحليل داده، طرح موضـوع و مســئله، يُيخيرى روند ثبت و ياسخ داورى مقاله در مجله

\section{تشكر و قدردانى}

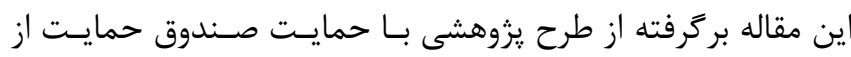

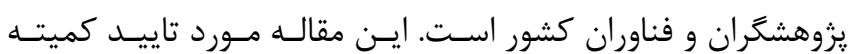

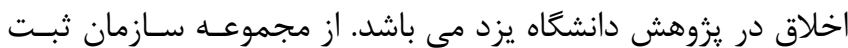
احوال ايران براى در اختيار دادن دادههاى مرك قدردانى مى شود.
يزوهشهاى خود نظر بر افزايش سرطان دهانه رحم و تخمدان براى

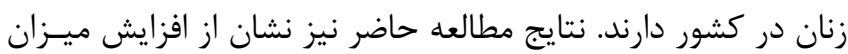

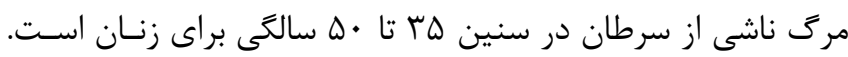
در مطالعهاى كه در مورد مركى ناشى از سرطان در ارويا انجـام شـد، ندان.

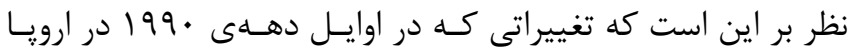

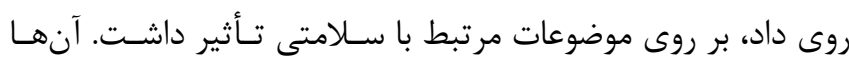

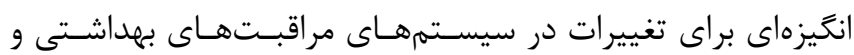

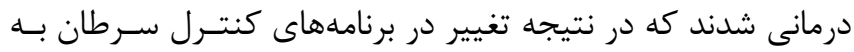

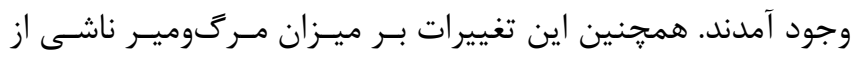

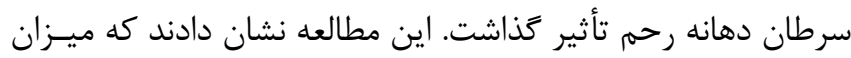
مركَومير ناشى از اين نوع سرطان در اكثر كشـورهاى موردمطالعـهـ

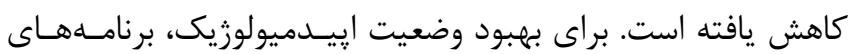

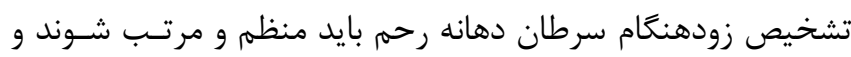

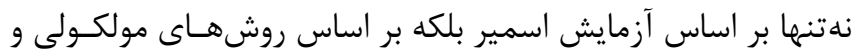

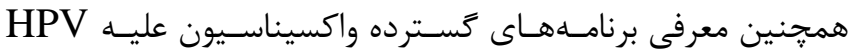

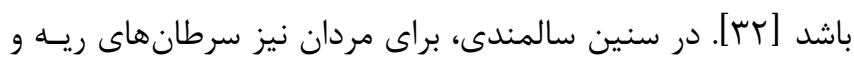

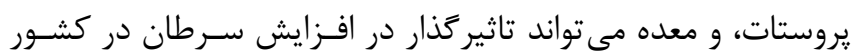

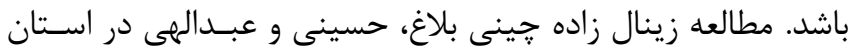

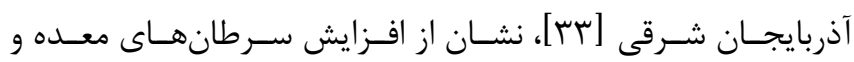

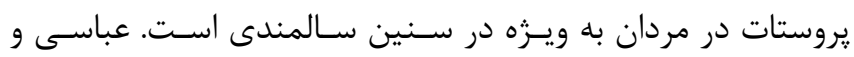

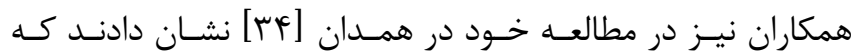
افزايش سرطان ريه در مردان در سنين سالمندى بيشتر بوده اسـت دئ دان

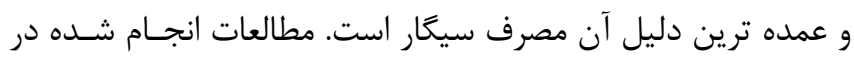

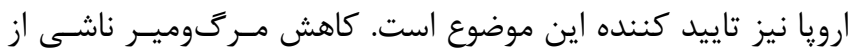

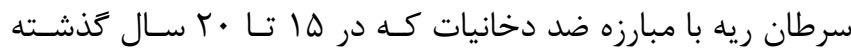

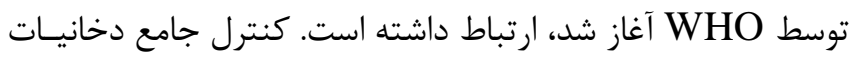

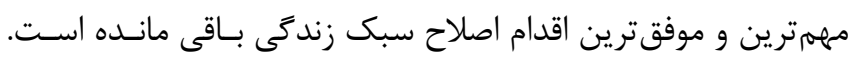

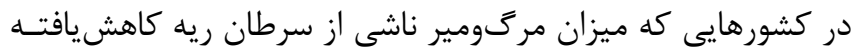

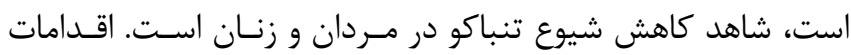

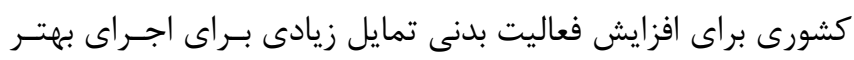

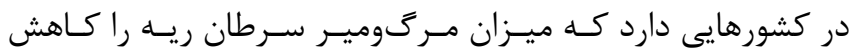

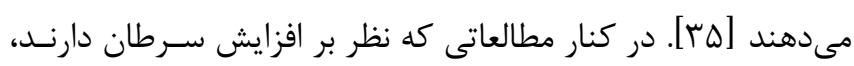

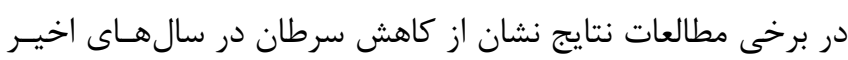

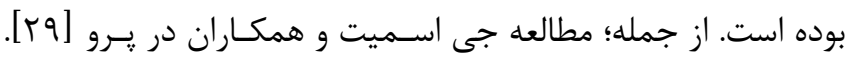

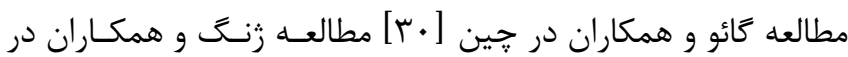

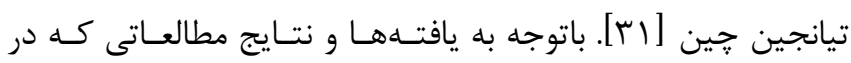




\section{منابع}

1. M.H. Wahdan. The epidemiological transition. Eastern Mediterranean Health Journal 1996; 2: 8-20

2. WHO. Global health estimates 2017 (deaths by cause, age, sex, by country and by region, 2000-2016; and life expectancy, 2000-2016. $1^{\text {st }}$ Edition, WHO: Geneva, 2018

3. Murray, Christopher \& Cristiana, Abbafati \& Abbas, et al. Five insights from the Global Burden of Disease Study 2019. Lancet 2020; 396: 1135-1159

4. Kyu HH Abate D Abate KH et al. Global, regional, and national disability-adjusted life-years (DALYs) for 359 diseases and injuries and healthy life expectancy (HALE) for 195 countries and territories, 1990-2017: a systematic analysis for the Global Burden of Disease Study 2017. Lancet 2018; 392: 1859-1922

5. Rudd K Johnson S Agesa K et al. Global, regional, and national sepsis incidence and mortality, 19902017. Lancet 2020; 395: 200-211

6. Theo Vos, Stephen S Lim, Cristiana Abbafati, et al. Global burden of 369 diseases and injuries in 204 countries and territories, 1990-2019. Lancet 2020; 396: 1204-1222

7. WHO. Noncommunicable diseases. $1^{\text {st }}$ Edition, WHO: Geneva, 2018

8. A. Friedman. Cancer as multifaceted disease. Mathematical Modelling of Natural Phenomena Journal 2012; 7: 3-28

9. Rebecca L. Siegel et al. Cancer Society. Cancer Facts \& Figures 2021. American Cancer Society Journal 2021: 71: 7-33

10. Stanaway J. Christopher J.L. Murray, Ashkan Afshin. Global, regional, and national comparative risk assessment of 84 behavioural, environmental and occupational, and metabolic risks or clusters of risks for 195 countries and territories, 1990-2017: a systematic analysis for the Global Burden of Disease Study 2017. Lancet 2018; 392:1923-94

11. C. Fitzmaurice, D. Abate, and N. Abbasi. Global, Regional, and National Cancer Incidence, Mortality, Years of Life Lost, Years Lived With Disability, and Disability-Adjusted Life-Years for 29 Cancer Groups, 1990 to 2017 A Systematic Analysis for the Global Burden of Disease Study, Journal of Clinical Oncology 2019; 12: 1749-1768

12. A. H. Zeinalzadeh, R. Hosseini, and L. Abdullahi. Survey of Epidemiology of Cancers in the Patients above 15 Years Old in East Azerbaijan Province, Iran
2013. Journal of Ardabil University of Medical Sciences 2015; 15: 46-55

13. Torkashvand Moradabadi M, Torkashvand Z. Epidemiological transition and the importance of social policy: mortality statistic in Ilam, Iran during 2011-2017. Payesh 2019; 18: 321-331 [Persian]

14. Naghavi M. Transition in Health Status in the Islamic Republic of Iran. Iranian Journal of Epidemiology 2006; 2: 45-57 [Persian]

15. Torkashvand Moradabadi M, Abbasi M. An Epidemiological study of mortality among elderly in Iran using Years of Life Lost (YLL) index during 2011 to 2017. Payesh 2020; 19: 85-97 [Persian]

16. Torkashvand Moradabadi, Mohammad, Sensitivity Analysis and Reexamination of the Techniques for Evaluating Adult Death Registration [On the basis of data of province of Iran], A thesis submitted to the graduate studies for the degree of Ph.D. in demography, Tehran University, 2017: DOI;13140/RG.2.2.34138.29123 [Persian]

17. Torkashvand Moradabadi, M., Falah, H. The Evaluation of the Death Registration Coverage in the Civil Registration Organization of Iran (Case Study of Yazd Province, 2016). Journal of Applied Sociology 2020; 31: 21-34

18. Koosheshi, M. \& Torkashvand Moradabadi, M. Investigating the Adaptation of Age Pattern of Mortality in Iran with Model Life Tables. Iranian Population Studies Journal 2018; 3: 39 - 63 [Persian]

19. Shokohmand, M. Investigating the Factors Affecting Late Birth and Death Registration in Ahvaz City, MA Thesis 2010, Islamic Azad University, Shoushtar Branch

20. Zanjani, H. Investigating Mortality in Iran from Census Data. Iranian Journal of Population Studies 1992; 3: 69-78 [Persian]

21. Zanjani, H. Evaluation of Location-Based Vital Event Information Record (Birth, Death and Internal Migration). Research Project, Bureau of Statistics and Population Data, Civil Registration Organization, Tehran 2013 [Persian]

22. M. S. Lima et al. Temporal trend of cancer mortality in a Brazilian state with a medium Human Development Index 1980-2018. Scientific Reports 2020; 10: $1-10$

23. F. K. Shadmani, F. Farzadfar, M. Yoosefi, K. Mansori, R. K. Shadman, and A. Haghdoost. Premature mortality of gastrointestinal cancer in Iran: 
Trends and projections 2001-2030. BioMed Central Cancer 2020; 20: 1-11

24. K. L. Yan et al. Rising incidence and incidencebased mortality of thyroid cancer in California, 20002017. Journal of Clin. Endocrinol. Metab 2020; 105: 1770-1777

25. L. Ventura et al. Estimates of cancer burden in Tuscany. Tumori Journal 2013; 99: 334-341

26. M. Fusco, R. De Angelis, G. Senatore, G. Zigon, and S. Rossi. Estimates of cancer burden in Campania. Tumori Journal 2013; 99: 374-381

27. S. Rosso et al. Estimates of cancer burden in Piedmont and Aosta Valley. Tumori Journal 2013; 99: 269-276

28. R. Galasso, R. Capocaccia, L. Del Riccio, L. Viviano, R. Matera, and S. Rossi. Estimates of cancer burden in Basilicata and Calabria. Tumori Journal 2013; 99: 390-398

29. fatemeh karimi, R. Entezar Mahdi, and H. R. KHalkhali. Estimation burden of breast cancer in West Azarbaijan province in year 2013. Urmia Medicine Jounal 2017; 28: 1-7

30. Z. Vafaeinezhad et al. Trends in Cervical Cancer Incidence in Iran According to National Cancer Registry. J Maz. University of Medical Sciences 2018; 28: $108-114$

31. Hoseini, $\mathrm{S}$ et al. Changes in prevalence and epidemiology of female genital cancers in northeastern Iran in a 35-year study. The Iranian Journal of Obstetrics, Gynecology and Infertility 2020; 22: 11-18
32. N. Khaltaev and S. Axelrod. Global lung cancer mortality trends and lifestyle modifications: Preliminary analysis. Chinical Medicine Journal 2020; 133: $1526-1532$

33. A. H. Zeinalzadeh, R. Hosseini, and L. Abdullahi. Survey of Epidemiology of Cancers in the Patients above 15 Years Old in East Azerbaijan Province, Iran 2013. J. Ardabil University Medical Scienesec 2015; 15: 46-55.

34. M. Abbasi, F. Moradi, F. Esna-Ashari, and M. A. Seifrabiei. Epidemiological and Pathological Study of Lung Cancer in Patients Referred to Ekbatan and Shahid Beheshti Hospitals in Hamadan during 2001 2016. Avicenna J. Clinimcal Medicine 2019; 25: 236243

35. N. Rajai et al. "National and subnational trends in incidence and mortality of lung cancer in Iran from 1990 to 2016. Asia. Pac. J. Clin. Oncol. 2020; 19: 1-8

36. J. S. Torres-Roman et al. Cervical cancer mortality in Peru: regional trend analysis from 2008-2017. BMC Public Health 2021; 21: 1-11

37. K. Gao and J. Wu. National trend of gastric cancer mortality in China (2003-2015): A population-based study. Cancer Commun 2019; 39: 1-5

38. W. Zheng et al. Trend analysis of lung cancer mortality and years of life lost (YLL) rate from 1999 to 2016 in Tianjin, China: Does the lung cancer burden in rural areas exceed that of urban areas? Thorac Cancer 2020; 28: 867-874 